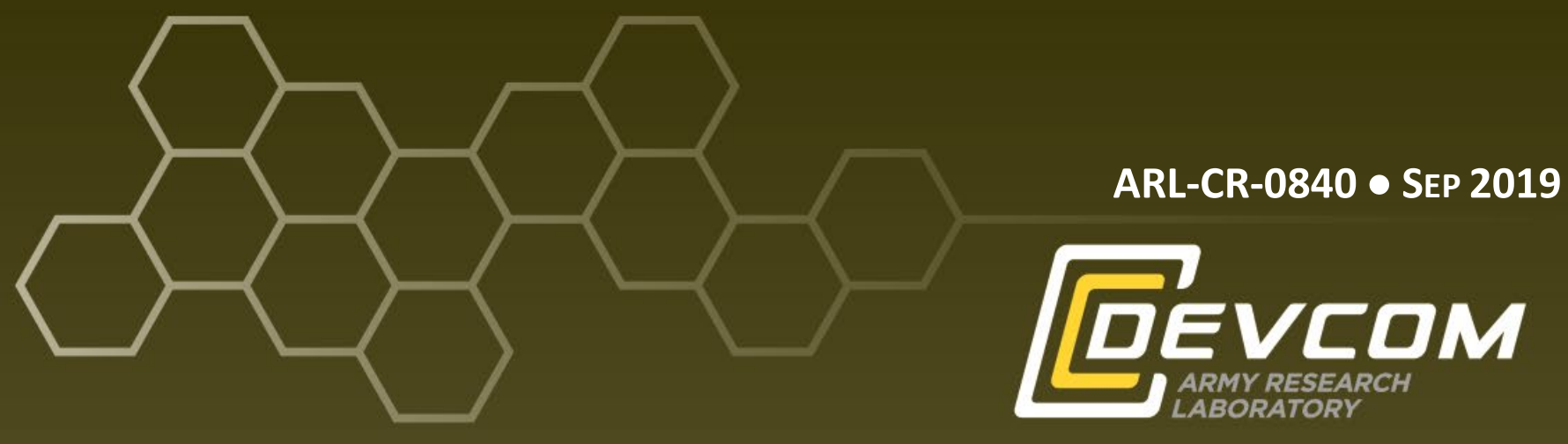

\title{
Methods for Determining the Initial State of a Projectile Equipped with Onboard Strapdown Sensors
}

by Bradley T Burchett Rose-Hulman Institute of Technology Terre Haute, Indiana

under contract W911NF-18-2-0171

Approved for public release; distribution is unlimited. 


\section{NOTICES}

\section{Disclaimers}

The findings in this report are not to be construed as an official Department of the Army position unless so designated by other authorized documents.

Citation of manufacturer's or trade names does not constitute an official endorsement or approval of the use thereof.

Destroy this report when it is no longer needed. Do not return it to the originator. 


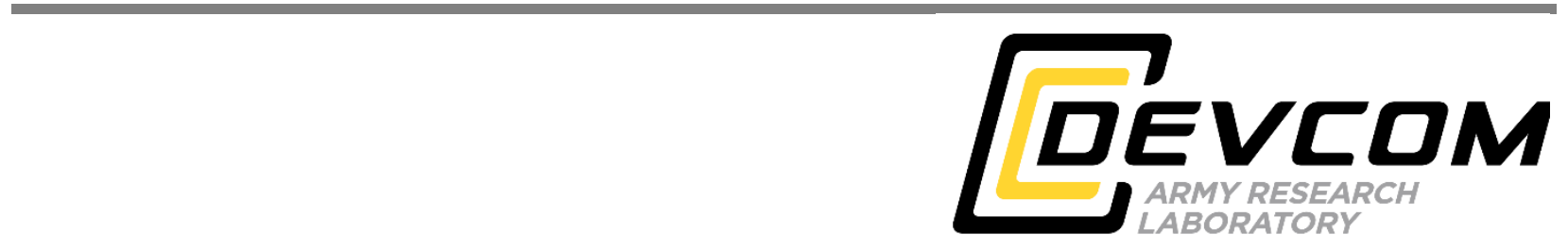

\section{Methods for Determining the Initial State of a Projectile Equipped with Onboard Strapdown Sensors}

Bradley T Burchett

Rose-Hulman Institute of Technology

Terre Haute, Indiana

under contract W911NF-18-2-0171

Approved for public release; distribution is unlimited. 
Public reporting burden for this collection of information is estimated to average 1 hour per response, including the time for reviewing instructions, searching existing data sources, gathering and maintaining the data needed, and completing and reviewing the collection information. Send comments regarding this burden estimate or any other aspect of this collection of information, including suggestions for reducing the burden, to Department of Defense, Washington Headquarters Services, Directorate for Information Operations and Reports (0704-0188), 1215 Jefferson Davis Highway, Suite 1204, Arlington, VA $22202-4302$. Respondents should be aware that notwithstanding any other provision of law, no person shall be subject to any penalty for failing to comply with a collection of information if it does not display a currently valid OMB control number.

PLEASE DO NOT RETURN YOUR FORM TO THE ABOVE ADDRESS.

\begin{tabular}{|l|l|}
\hline $\begin{array}{l}\text { 1. REPORT DATE (DD-MM-YYYY) } \\
\text { September } 2019\end{array}$ & $\begin{array}{l}\text { 2. REPORT TYPE } \\
\text { Contractor Report }\end{array}$ \\
\hline
\end{tabular}

4. TITLE AND SUBTITLE

Methods for Determining the Initial State of a Projectile Equipped with Onboard Strapdown Sensors

3. DATES COVERED (From - To)

04 June-16 August 2019

5a. CONTRACT NUMBER

W911NF-18-2-0171

5b. GRANT NUMBER

5c. PROGRAM ELEMENT NUMBER

5d. PROJECT NUMBER

6. AUTHOR(S)

5e. TASK NUMBER

5f. WORK UNIT NUMBER

8. PERFORMING ORGANIZATION REPORT NUMBER

7. PERFORMING ORGANIZATION NAME(S) AND ADDRESS(ES)

Rose-Hulman Institute of Technology

5500 Wabash Ave

Terre Haute, IN 47803

9. SPONSORING/MONITORING AGENCY NAME(S) AND ADDRESS(ES)

10. SPONSOR/MONITOR'S ACRONYM(S)

CCDC Army Research Laboratory

ATTN: FCDD-RLW-LE

Aberdeen Proving Ground, MD 21005

11. SPONSOR/MONITOR'S REPORT NUMBER(S)

ARL-CR-0840

\section{DISTRIBUTION/AVAILABILITY STATEMENT}

Approved for public release; distribution is unlimited.

\section{SUPPLEMENTARY NOTES}

ORCID ID: Bradley T Burchett, 0000-0002-1934-0537

\section{ABSTRACT}

Projectile aerodynamics for long-range and indirect-fire shots may be characterized using onboard magnetometer, gyroscope, and accelerometer sensors. Previous researchers have developed ways to match projectile aerodynamic parameters to the collected measurements; however, accurate reconstruction of the projectile trajectory was hindered by the ability to accurately estimate projectile initial states. In this work, we demonstrate the application of several models to better match the trajectory and sequentially update estimates of the initial projectile state. The final product is a set of algorithms that renders accurate initial-condition estimates that can then be merged with an existing high-fidelity ballistics code in order to provide accurate reconstruction of the projectile trajectory. Models for both 6- and 7-degree-of-freedom projectiles are demonstrated. The algorithms are exercised on actual and virtual data sets, and convergence to realistic initial conditions, parameters, and trajectories is reliable even in the presence of unmodeled sensor errors.

\section{SUBJECT TERMS}

onboard sensors, projectile aerodynamics, trajectory reconstruction, initial conditions, dual spin

\begin{tabular}{|c|c|c|}
\hline \multicolumn{3}{|c|}{ 16. SECURITY CLASSIFICATION OF: } \\
\hline a. REPORT & b. ABSTRACT & c. THIS PAGE \\
\hline Unclassified & Unclassified & Unclassified \\
\hline
\end{tabular}

\begin{tabular}{|c|c|}
$\begin{array}{l}\text { 17. LIMITATION } \\
\text { OF ABSTRACT }\end{array}$ & $\begin{array}{c}\text { 18. NUMBER } \\
\text { OF PAGES }\end{array}$ \\
UU & 52 \\
\hline
\end{tabular}

19a. NAME OF RESPONSIBLE PERSON

Bradley T Burchett

19b. TELEPHONE NUMBER (Include area code)

(812) 877-8929

Standard Form 298 (Rev, 8/98) Prescribed by ANSI Std. Z39.18 
List of Figures $\quad$ iv

List of Tables $\quad$ iv

$\begin{array}{ll}\text { 1. Introduction } & 1\end{array}$

2. Three- and 6-DOF Models of Projectile Flight 3

3. Measurement Models for Onboard Sensors 4

4. Derivatives of Onboard Sensor Measurements with Respect to the State

5. Methods for Estimating Initial Position and Euler Angles

6. Finding Initial Conditions by Merging the UKF with High-Fidelity Dynamics

7. Finding Initial Conditions by Forward Integration of a 7-DOF Linear Model

7.1 Solution for the Linear 7-DOF Model 21

7.2 Differentiating the Solution with Respect to the Initial Conditions 22

7.3 Finding Best Model Parameters through the Marquardt Algorithm 24

8. Sensitivities of a Nonlinear 6-DOF Model wrt Initial Conditions 27

8.1 Results with Virtual Data 33

8.2 Results with Actual Data 34

9. Conclusion 40

10. References 42

List of Symbols, Abbreviations, and Acronyms 44

$\begin{array}{ll}\text { Distribution List } & 46\end{array}$ 


\section{List of Figures}

Fig. 1 The HMA 83-mm dual spin vehicle ..................................................2

Fig. 2 Trajectory matching using a point-mass model with analytic derivatives on the radar data only....................................................................... 12

Fig. 3 Flow chart for applying UKF to high-fidelity simulation "HMA"........13

Fig. 4 Second method's trajectory matching using the UKF with high-fidelity dynamics; backward integration from $0.1 \mathrm{~s}$ ignores the measurements .15

Fig. 5 Trajectory matching in second method using the UKF with high-fidelity dynamics; backward integration from $0.1 \mathrm{~s}$ ignores the measurements .16

Fig. 6 Trajectory matching using the UKF with high-fidelity dynamics; backward integration from $0.1 \mathrm{~s}$ ignores the measurements.....

Fig. 7 Trajectory matching using the 7-DOF linear model with Marquardt algorithm

Fig. 8 High-fidelity model predictions of the onboard sensor signals after 15 iterations of NLLS refinements to ICs and linear aerodynamic

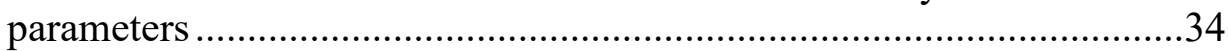

Fig. 9 Model predictions and actual measurements for HMA Flight 8 data.....35

Fig. 10 In repeated process, model predictions and actual measurements for HMA Flight 8 data

Fig. 11 Process repeated again, model predictions and actual measurements for HMA Flight 8 data

\section{List of Tables}

Table 1 Initial-condition estimates rendered by several methods applied to virtual data

Table 2 IC estimates from reducing actual HMA data ......................................36

Table 3 Initial and final aerodynamic parameter estimates .................................37

Table 4 IC estimates from reducing actual HMA data, second phase of flight ...38

Table 5 Initial and final aerodynamic parameter estimates, second phase of

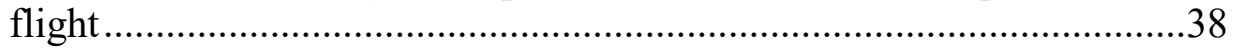

Table 6 IC estimates from reducing actual HMA data, third phase of flight.......40

Table 7 Initial and final aerodynamic parameter estimates, third phase of flight40 


\section{Introduction}

State-of-the-art methods for estimating the aerodynamics of projectiles include wind-tunnel tests, spark-range firings, and free flight with onboard sensors. For the sake of accurate aerodynamic models, free-flight tests are preferred over wind tunnel. Spark range tests are limited to short range and flat fire. Thus, for projectiles with long-range and indirect fire, free flight with onboard sensors remains the best option.

Measurements for such tests are generally limited to onboard magnetometer, gyroscope, and accelerometer readings plus externally observed radar azimuth, elevation, and range rate. Unlike spark-range shadowgraphs, none of these measurements directly correlate to the projectile's absolute position and orientation in space. Moreover, gyroscope and accelerometer readings are strongly correlated to projectile angular rates and linear accelerations as opposed to position and orientation.

In light of these observations, the free-flight trajectory may be modelled by forward integration of a dynamic model, and output error methods may be used to estimate aerodynamic coefficients in order to match the data in a least-squares sense. However, determining the initial projectile state for best trajectory match appears to be an open problem. ${ }^{1-6}$

For several decades, reduction of spark-range data was handled by matching a linearized trajectory model to the data. ${ }^{7}$ Models of progressively higher fidelity were used in a deliberate sequence. Then, Chapman and Kirk ${ }^{8,9}$ introduced a method to fit nonlinear 6 degree-of-freedom (DOF) models directly to spark-range data. Their method is widely used to date with a few modest enhancements. Hathaway and Whyte ${ }^{10}$ extended the Chapman-Kirk method to include onboard sensor measurements. Their work resulted in the Extending Telemetry Reduction to Aerodynamic Coefficients and Trajectory Reconstruction (EXTRACTR) software licensed by Arrow Tech Associates. Davis et al. ${ }^{11}$ demonstrated the use of EXTRACTR in a comprehensive set of tests on 155-mm M483A1 projectiles with instrumented fuses developed at the US Army Research Laboratory. More recently, Fresconi and Harkins applied the onboard-sensor technique to projectiles equipped with control mechanisms. ${ }^{12,13}$ The second of their papers introduced asymmetric aerodynamics including a side moment into the model and used an onboard solar yawsonde to resolve the correct sense of projectile coning motion. Davis et al. ${ }^{14}$ instrumented an 81-mm M879 mortar. Some data gathered from their experiment were used to test the algorithms in Burchett. ${ }^{5}$ More sophisticated measurements can now be incorporated, and modern personal computers allow even large data sets to 
be processed in a few minutes at one's desk. We demonstrate three versions of the Chapman-Kirk method in Sections 5, 7, and 8 of this report, and a related recursive method in Section 6. The Chapman-Kirk method still requires good initial guesses as it suffers from a small region of convergence. Sections 5 and 7 attempt to address this issue by fitting simpler models and restricting the measurements to a relevant subset to obtain good guesses for a subset of the states.

This work is primarily targeted at developing an algorithm to extract aerodynamic parameters for the High Maneuverability Airframe (HMA) depicted in Fig. 1. HMA is a 7-DOF vehicle with a highly instrumented forward section with four independently controlled canards. The tail section has eight canted fins to induce spin stability but is isolated from the nose by a smooth bearing. The roll and roll rate of the tail are not measured. The extra DOF and lack of measurement present unique challenges, which are discussed in Section 6.

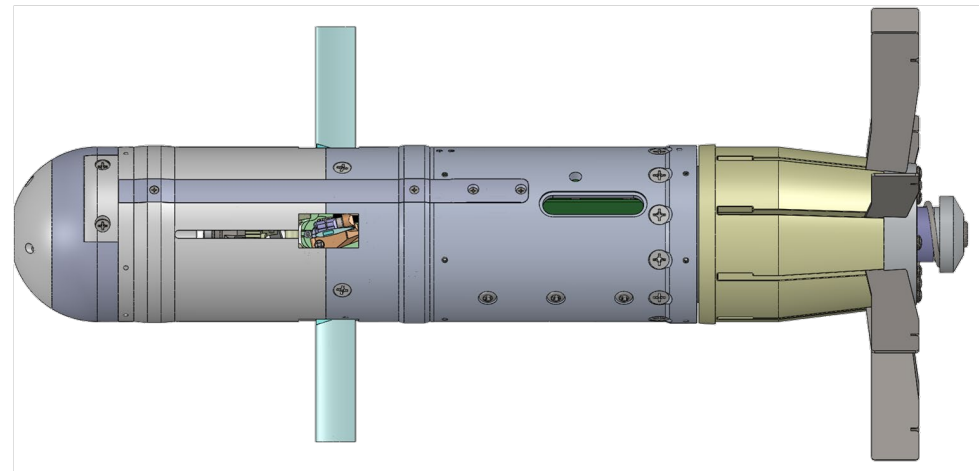

Fig. 1 The HMA 83-mm dual spin vehicle

In this work, we will review several methods for estimating the initial-state vector. Various levels of model fidelity are used to find subsets of initial states. Three methods matching full models will be explored. First, the initial Euler angles can be found from the initial magnetometer readings through numerical root finding. Second, the initial-position vector can be found by propagating a point-mass model and sensitivities forward in time. These sensitivities can then be used with the radar measurements in a Marquardt algorithm to render an accurate estimate of the initial position vector. Third, the application of an unscented Kalman filter (UKF) to 7-DOF high-fidelity dynamics allows for accurate estimates of projectile initial velocities and angular rates. Fourth, a 7-DOF linear model is leveraged to estimate the full initial state by propagating sensitivities forward in time in parallel with trajectory predictions. Finally, sensitivities of states to initial states are found for a 6-DOF nonlinear model. These are incorporated with a high-fidelity simulation to iterate both initial states and aerodynamic parameters simultaneously. 


\section{Three- and 6-DOF Models of Projectile Flight}

Two models most frequently used to model projectile flight are presented here. The 3-DOF or point-mass model may be used to predict a projectile's trajectory with respect to the ground when only the position of the projectile's center of gravity (CG) is of interest. Such a model may be used for initial estimates of the total drag coefficient, and initial position of the projectile CG. The following six equations, 1a-1f, describe the position and velocity of the projectile CG with respect to a flat, nonrotating earth in a ground-fixed frame:

$$
\begin{gathered}
\dot{x}=V_{x}, \\
\dot{y}=V_{y}, \\
\dot{z}=V_{z}, \\
\dot{V}_{x}=-\hat{C}_{x} \sqrt{V_{x}^{2}+V_{y}^{2}+V_{z}^{2}} \cdot V_{x}, \\
\dot{V}_{y}=-\hat{C}_{x} \sqrt{V_{x}^{2}+V_{y}^{2}+V_{z}^{2}} \cdot V_{y},
\end{gathered}
$$

and

$$
\dot{V}_{z}=-\hat{C}_{x} \sqrt{V_{x}^{2}+V_{y}^{2}+V_{z}^{2}} \cdot V_{z}+g
$$

where the $+g$ term indicates that altitude is positive down, $\hat{C}_{x}$ is a dimensional drag coefficient [1/length] and lift is considered negligible.

The standard nonlinear 6-DOF equations of motion ${ }^{4}$ shown in Eqs. 2a-2d, are used when projectile position and orientation predictions are required. This level of fidelity is needed when reducing onboard sensors since the measurements are functions of projectile orientation, angular rates, and linear accelerations:

$$
\begin{gathered}
\left\{\begin{array}{c}
\dot{x} \\
\dot{y} \\
\dot{z}
\end{array}\right\}=T_{b 2 i}^{T}\left\{\begin{array}{c}
u \\
v \\
w
\end{array}\right\}=f(\phi, \theta, \psi, u, v, w), \\
\left\{\begin{array}{c}
\dot{\phi} \\
\dot{\theta} \\
\dot{\psi}
\end{array}\right\}=T_{b 2 e}\left\{\begin{array}{l}
p \\
q \\
r
\end{array}\right\}=g(\phi, \theta, p, q, r),
\end{gathered}
$$




$$
\left\{\begin{array}{c}
\dot{u} \\
\dot{v} \\
\dot{w}
\end{array}\right\}=\left\{\begin{array}{l}
X / m \\
Y / m \\
Z / m
\end{array}\right\}-\mathbf{S}\left\{\begin{array}{c}
u \\
v \\
w
\end{array}\right\}=h(\phi, \theta, \psi, u, v, w, p, q, r)
$$

and

$$
\left\{\begin{array}{l}
\dot{p} \\
\dot{q} \\
\dot{r}
\end{array}\right\}=[\mathbf{I}]^{-1}\left[\left(\begin{array}{c}
L \\
M \\
N
\end{array}\right\}-\mathbf{S}[\mathbf{I}]\left\{\begin{array}{l}
p \\
q \\
r
\end{array}\right\}\right]=k(p, q, r)
$$

where

$$
\begin{array}{cccc}
c_{\theta} c_{\psi} & c_{\theta} s_{\psi} & -s_{\theta} \\
T_{b 2 i}=\left[\begin{array}{ccc}
s_{\phi} s_{\theta} c_{\psi}-c_{\phi} s_{\psi} & s_{\phi} s_{\theta} s_{\psi}+c_{\phi} c_{\psi} & s_{\phi} c_{\theta} \\
c_{\phi} s_{\theta} c_{\psi}+s_{\phi} s_{\psi} & c_{\phi} s_{\theta} s_{\psi}-s_{\phi} c_{\psi} & c_{\phi} c_{\theta}
\end{array}\right] \\
T_{b 2 e}=\left[\begin{array}{ccc}
1 & s_{\phi} t_{\theta} & -c_{\phi} t_{\theta} \\
0 & c_{\phi} & -s_{\phi} \\
0 & s_{\phi} / c_{\theta} & c_{\phi} / c_{\theta}
\end{array}\right] & \mathbf{S}=\left[\begin{array}{ccc}
0 & -r & q \\
r & 0 & -p \\
-q & p & 0
\end{array}\right] .
\end{array}
$$

Some terms of interest from the force $\left\{\begin{array}{llll}X & Y & Z\end{array}\right\}$ and moment $\left\{\begin{array}{lll}L & M & N\end{array}\right\}$ vectors are unpacked in a subsequent section. (For a more complete treatment, please see Burchett, ${ }^{1} \mathrm{McCoy},{ }^{4}$ or Fresconi et al. ${ }^{6}$ )

\section{Measurement Models for Onboard Sensors}

Four types of measurements are gathered during free-flight testing with onboard sensors. These are 1) external radar radial velocity, elevation and azimuth, 2) strapdown gyroscope, 3) onboard magnetometer, and 4) strapdown accelerometer readings. First, we define the external radar measurement vector $\left\{\begin{array}{lll}R_{V} & R_{\theta} & R_{\psi}\end{array}\right\}$ :

$$
\begin{gathered}
R_{V}=\frac{\dot{x} \delta x+\dot{y} \delta y+\dot{z} \delta z}{\sqrt{\delta x^{2}+\delta y^{2}+\delta z^{2}}}, \\
R_{\theta}=\tan ^{-1}(\delta z / \delta x)
\end{gathered}
$$

and

$$
R_{\psi}=\tan ^{-1}(\delta y / \delta x)
$$

where 


$$
\begin{aligned}
& \delta x=x-x_{A} \\
& \delta y=y-y_{A}
\end{aligned}
$$

and

$$
\delta z=z-z_{A}
$$

And, $\left\{\begin{array}{lll}x_{A} & y_{A} & z_{A}\end{array}\right\}$ is the radar antenna position in the gun-tube frame.

A strapdown gyroscope measures the projectile angular rates $\left\{\begin{array}{lll}p & q & r\end{array}\right\}$, in the body frame. The onboard magnetometer measures the earth's magnetic field in the body frame:

$$
\left\{\begin{array}{l}
\tilde{m}_{E x} \\
\tilde{m}_{E y} \\
\tilde{m}_{E z}
\end{array}\right\}=T_{b 2 i}\left[\begin{array}{ccc}
c_{\alpha} & s_{\alpha} & 0 \\
-s_{\alpha} & c_{\alpha} & 0 \\
0 & 0 & 1
\end{array}\right]\left\{\begin{array}{l}
m_{E x} \\
m_{E y} \\
m_{E z}
\end{array}\right\}
$$

where $\alpha$ is the launch azimuth clockwise from true north.

The mapping from projectile states to accelerometer measurements is given by Eq. 5:

$$
\left\{\begin{array}{l}
\tilde{a}_{x} \\
\tilde{a}_{y} \\
\tilde{a}_{z}
\end{array}\right\}=\left(\left\{\begin{array}{c}
\dot{u} \\
\dot{v} \\
\dot{w}
\end{array}\right\}+\mathbf{S}\left\{\begin{array}{c}
u \\
v \\
w
\end{array}\right\}-T_{b 2 i}\left\{\begin{array}{l}
0 \\
0 \\
g
\end{array}\right\}+\mathbf{S} \cdot \mathbf{S} \cdot \mathbf{R}_{\mathbf{A}}+\dot{\mathbf{S}} \cdot \mathbf{R}_{\mathbf{A}}\right)
$$

$\mathbf{S}$ and $\dot{\mathbf{S}}$ are skew matrices contrived to perform cross products

$$
\mathbf{S}=\left[\begin{array}{ccc}
0 & -r & q \\
r & 0 & -p \\
-q & p & 0
\end{array}\right] \quad \dot{\mathbf{S}}=\left[\begin{array}{ccc}
0 & -\dot{r} & \dot{q} \\
\dot{r} & 0 & -\dot{p} \\
-\dot{q} & \dot{p} & 0
\end{array}\right]
$$

and $\mathbf{R}_{\mathbf{A}}$ is the vector from projectile $\mathrm{CG}$ to accelerometer position in the body frame. The full measurement vector can then be assembled as

$$
\mathbf{Y}=\left[\begin{array}{llllllllllll}
\tilde{m}_{E x} & \tilde{m}_{E y} & \tilde{m}_{E z} & p & q & r & \tilde{a}_{x} & \tilde{a}_{y} & \tilde{a}_{z} & R_{V} & R_{\theta} & R_{\psi}
\end{array}\right]
$$

\section{Derivatives of Onboard Sensor Measurements with Respect to the State}

In the subsequent sections, several gradient-based searches are proposed to match a model trajectory to the onboard sensor measurements. A fundamental concept 
behind each of these methods is that the sensitivities of measurements with respect to the adjustable parameters $\partial \mathbf{Y} / \partial \boldsymbol{\vartheta}$ may be found by applying the chain rule

$$
\frac{\partial \mathbf{Y}}{\partial \boldsymbol{\vartheta}}=\frac{\partial \mathbf{Y}}{\partial \mathbf{X}} \frac{\partial \mathbf{X}}{\partial \boldsymbol{\vartheta}}
$$

where the parameter vector $\boldsymbol{\vartheta}$ may contain both aerodynamic coefficients, and initial conditions. Due to the nonlinear mapping from model states to measurement predictions, nontrivial sensitivities of measurements with respect to (wrt) projectile states, $\partial \mathbf{Y} / \partial \mathbf{X}$, must be found.

The $12 \times 12$ derivative matrix will be filled in blocks as

$$
\frac{\partial \mathbf{Y}}{\partial \mathbf{X}}=\left[\begin{array}{cccc}
0 & \frac{\partial \mathbf{m}}{\partial \Theta} & 0 & 0 \\
0 & \frac{\partial \boldsymbol{\omega}}{\partial \boldsymbol{\Theta}} & 0 & \frac{\partial \boldsymbol{\omega}}{\partial \boldsymbol{\omega}_{n r}} \\
0 & \frac{\partial \mathbf{a}}{\partial \boldsymbol{\Theta}} & \frac{\partial \mathbf{a}}{\partial \mathbf{U}} & \frac{\partial \mathbf{a}}{\partial \boldsymbol{\omega}} \\
\frac{\partial \mathbf{R}}{\partial \mathbf{X}} & \frac{\partial \mathbf{R}}{\partial \Theta} & \frac{\partial \mathbf{R}}{\partial \mathbf{U}} & 0
\end{array}\right]
$$

where the vectors $\mathbf{m}=\left\{\begin{array}{lllll}\tilde{m}_{E x} & \tilde{m}_{E y} & \tilde{m}_{E z}\end{array}\right\}, \quad \mathbf{a}=\left\{\begin{array}{llll}\tilde{a}_{x} & \tilde{a}_{y} & \tilde{a}_{z}\end{array}\right\}, \quad \mathbf{R}=$ $\left\{\begin{array}{llll}R_{V} & R_{\theta} & R_{\psi}\end{array}\right\}, \quad \boldsymbol{\omega}=\left\{\begin{array}{lll}p & q & r\end{array}\right\}, \quad \boldsymbol{\Theta}=\left\{\begin{array}{llll}\phi & \theta & \psi\end{array}\right\}, \quad \mathbf{X}=\left\{\begin{array}{lll}x & y & z\end{array}\right\}, \quad \mathbf{U}=$ $\left\{\begin{array}{lll}u & v & w\end{array}\right\}$ are used for shorthand.

Then

$$
\frac{\partial \mathbf{m}}{\partial \boldsymbol{\Theta}}=\left[\begin{array}{lll}
\frac{\partial \mathbf{m}}{\partial \phi} & \frac{\partial \mathbf{m}}{\partial \theta} & \frac{\partial \mathbf{m}}{\partial \psi}
\end{array}\right]
$$

and

$$
\frac{\partial \mathbf{m}}{\partial \phi}=\frac{\partial T_{b 2 i}}{\partial \phi}\left[\begin{array}{ccc}
c_{\alpha} & s_{\alpha} & 0 \\
-s_{\alpha} & c_{\alpha} & 0 \\
0 & 0 & 1
\end{array}\right]\left\{\begin{array}{l}
m_{E x} \\
m_{E y} \\
m_{E z}
\end{array}\right\},
$$

and so on.

Since a linear model may be defined in a no-roll frame, gyroscope measurements are sensitive to the Euler angles due to the conversion from no-roll to body frame. Thus 


$$
\frac{\partial \boldsymbol{\omega}}{\partial \boldsymbol{\omega}_{n r}}=\left[\begin{array}{ccc}
1 & 0 & 0 \\
0 & c_{\phi} & s_{\phi} \\
0 & -s_{\phi} & c_{\phi}
\end{array}\right]
$$

and

$$
\frac{\partial \boldsymbol{\omega}}{\partial \boldsymbol{\phi}}=\left[\begin{array}{ccc}
0 & 0 & 0 \\
0 & -s_{\phi} & c_{\phi} \\
0 & -c_{\phi} & -s_{\phi}
\end{array}\right]\left\{\begin{array}{l}
p \\
q \\
r
\end{array}\right\}_{n r}
$$

however, the right-hand side (RHS) of Eqs. 9 and 10 become identity and zero respectively when using a nonlinear model with angular rates predicted in the body frame. Also

$$
\frac{\partial \mathbf{R}}{\partial \mathbf{X}}=\left[\begin{array}{ccc}
\frac{\partial R_{V}}{\partial x} & \frac{\partial R_{V}}{\partial y} & \frac{\partial R_{V}}{\partial z} \\
\frac{\partial R_{\theta}}{\partial x} & 0 & \frac{\partial R_{\theta}}{\partial z} \\
\frac{\partial R_{\psi}}{\partial x} & \frac{\partial R_{\psi}}{\partial y} & 0
\end{array}\right]
$$

and

$$
\begin{array}{ll}
\frac{\partial R_{\theta}}{\partial x}=\frac{-\delta z}{\left(\delta x^{2}\left(\frac{\delta z^{2}}{\delta x^{2}}+1\right)\right)} & \frac{\partial R_{\theta}}{\partial z}=\frac{1}{\left(\delta x\left(\frac{\delta z^{2}}{\delta x^{2}}+1\right)\right)} \\
\frac{\partial R_{\psi}}{\partial x}=\frac{-\delta y}{\left(\delta x^{2}\left(\frac{\delta y^{2}}{\delta x^{2}}+1\right)\right)} & \frac{\partial R_{\psi}}{\partial y}=\frac{1}{\left(\delta x\left(\frac{\delta y^{2}}{\delta x^{2}}+1\right)\right)} .
\end{array}
$$

The radar radial velocity is sensitive to the Euler angles as

$$
\frac{\partial R_{V}}{\partial \theta}=\left[\begin{array}{lll}
\frac{\partial V_{R}}{\partial \dot{x}} & \frac{\partial V_{R}}{\partial \dot{y}} & \frac{\partial V_{R}}{\partial \dot{z}}
\end{array}\right] \cdot \frac{\partial T_{b 2 i}^{T}}{\partial \theta}\left\{\begin{array}{c}
u \\
v \\
W
\end{array}\right\}
$$

and 


$$
\frac{\partial R_{V}}{\partial \psi}=\left[\begin{array}{lll}
\frac{\partial V_{R}}{\partial \dot{x}} & \frac{\partial V_{R}}{\partial \dot{y}} & \frac{\partial V_{R}}{\partial \dot{z}}
\end{array}\right] \cdot \frac{\partial T_{b 2 i}^{T}}{\partial \psi}\left\{\begin{array}{c}
u \\
v \\
W
\end{array}\right\}
$$

The radar radial velocity is sensitive to the body-frame velocities as

$$
\frac{\partial R_{V}}{\partial \mathbf{U}}=\left[\begin{array}{lll}
\frac{\partial V_{R}}{\partial \dot{x}} & \frac{\partial V_{R}}{\partial \dot{y}} & \frac{\partial V_{R}}{\partial \dot{z}}
\end{array}\right] \cdot T_{b 2 i}^{T}\left[\begin{array}{ccc}
1 & 0 & 0 \\
0 & c_{\phi} & s_{\phi} \\
0 & -s_{\phi} & c_{\phi}
\end{array}\right]
$$

with the last factor applied only when the states are predicted in a no-roll frame. The vector of sensitivities wrt the ground-frame velocities is found by

$$
\left[\begin{array}{lll}
\partial \dot{x} & \frac{\partial V_{R}}{\partial \dot{y}} & \frac{\partial V_{R}}{\partial \dot{z}}
\end{array}\right]=\frac{\left[\begin{array}{lll}
\delta x & \delta y & \delta z
\end{array}\right]}{\sqrt{\delta x^{2}+\delta y^{2}+\delta z^{2}}} .
$$

Finally,

$$
\frac{\partial \mathbf{a}}{\partial \boldsymbol{\Theta}}=\left[\begin{array}{lll}
\frac{\partial \mathbf{a}}{\partial \phi} & \frac{\partial \mathbf{a}}{\partial \theta} & \frac{\partial \mathbf{a}}{\partial \psi}
\end{array}\right]
$$

where

$$
\frac{\partial \mathbf{a}}{\partial \phi}=\frac{\partial}{\partial \phi}\left[\begin{array}{ccc}
c_{\theta} c_{\psi} & c_{\theta} s_{\psi} & -s_{\theta} \\
s_{\phi} s_{\theta} c_{\psi}-c_{\phi} s_{\psi} & s_{\phi} s_{\theta} s_{\psi}+c_{\phi} c_{\psi} & s_{\phi} c_{\theta} \\
c_{\phi} s_{\theta} c_{\psi}+s_{\phi} s_{\psi} & c_{\phi} s_{\theta} s_{\psi}-s_{\phi} c_{\psi} & c_{\phi} c_{\theta}
\end{array}\right]\left\{\begin{array}{l}
0 \\
0 \\
g
\end{array}\right\}
$$

and so on;

$$
\frac{\partial \mathbf{a}}{\partial \boldsymbol{\omega}}=\left[\begin{array}{lll}
\frac{\partial \mathbf{a}}{\partial p} & \frac{\partial \mathbf{a}}{\partial q} & \frac{\partial \mathbf{a}}{\partial r}
\end{array}\right]
$$

where

$$
\frac{\partial \mathbf{a}}{\partial p}=\frac{\partial \mathbf{S}}{\partial p}\left\{\begin{array}{l}
u \\
v \\
w
\end{array}\right\}
$$

and so forth, and

$$
\frac{\partial \mathbf{a}}{\partial \mathbf{U}}=\mathbf{S}
$$




\section{Methods for Estimating Initial Position and Euler Angles}

Exact initial values of the Euler angles may be found by solving Eq. 4 using the initial magnetometer measurements. Since an analytic solution is not possible, a Newton method or Marquardt method may be used to reduce the prediction residuals below a certain threshold. Applying such a method for 15 Marquardt iterations results in the estimates shown in Table 1 under the "Estimate (static)" column. These estimates were used to seed the 7-DOF linear model iteration described in Section 7, since the initial Euler angles are not adjusted in the 7-DOF linear method.

Examining Eqs. 3a-3f, each sample of three radar measurements is dependent upon six quantities $(x, y, z, \dot{x}, \dot{y}, \dot{z})$. Thus, unlike the magnetometer observation, initial values of projectile position and velocity cannot be found from a static solution at the initial measurement. In other words, we cannot solve for six unknowns using only the three equations $(3 \mathrm{a}-3 \mathrm{c})$. Thus, a model must be fit to the history of radar measurements, rather than just the initial point. The simplest model for this motion is a point-mass no-lift model in 3-D as shown in Eqs. 1a-1f.

Integrating Eqs. 1a-1f forward and backward in time with Eqs. 3a-3c as the measurement model and using an UKF proved vain as downrange and altitude state estimates did not converge to the true values. This seems to indicate the point-mass model is unobservable when coupled with the radar measurements only.

Thus, a gradient-based search using the entire batch of trajectory predictions, and an analog Jacobian matrix is sought. Terms in the Jacobian can once again be found using

$$
\frac{\partial \mathbf{Y}}{\partial \mathbf{X}_{0}}=\frac{\partial \mathbf{Y}}{\partial \mathbf{X}} \frac{\partial \mathbf{X}}{\partial \mathbf{X}_{0}}
$$

and the first term on the RHS was defined in Eq. 11. The second term on the RHS may be found by numerical integration of a set of costates, which are found by

$$
\frac{d}{d t}\left(\frac{\partial \mathbf{X}}{\partial \mathbf{X}_{0}}\right)=\frac{\partial \dot{\mathbf{X}}}{\partial \mathbf{X}} \frac{\partial \mathbf{X}}{\partial \mathbf{X}_{0}} .
$$

For the rigid-body modes, any change in the launch position will be directly added to the trajectory coordinates for the entire flight:

$$
\frac{\partial x}{\partial x_{0}}=\frac{\partial y}{\partial y_{0}}=\frac{\partial z}{\partial z_{0}}=1 .
$$


Sensitivities of position states wrt initial velocities are, in turn

$$
\begin{gathered}
\frac{\partial}{\partial V_{x 0}}(\dot{x})=\frac{\partial V_{x}}{\partial V_{x 0}}, \\
\frac{\partial}{\partial V_{y 0}}(\dot{y})=\frac{\partial V_{y}}{\partial V_{y 0}},
\end{gathered}
$$

and

$$
\frac{\partial}{\partial V_{z 0}}(\dot{z})=\frac{\partial V_{z}}{\partial V_{z 0}}
$$

And, the terms on the RHS of Eq. $21 \mathrm{~d}-21 \mathrm{f}$ are found by propagating the following set of equations forward in time:

$$
\begin{aligned}
& \frac{d}{d t}\left(\frac{\partial V_{x}}{\partial V_{x 0}}\right)=-\hat{C}_{x} \sqrt{V_{x}^{2}+V_{y}^{2}+V_{z}^{2}} \cdot \frac{\partial V_{x}}{\partial V_{x 0}} \\
& -\hat{C}_{x}\left(V_{x}^{2}+V_{y}^{2}+V_{z}^{2}\right)^{-\frac{1}{2}} V_{x}^{2} \cdot \frac{\partial V_{x}}{\partial V_{x 0}} \text {, } \\
& \frac{d}{d t}\left(\frac{\partial V_{y}}{\partial V_{y 0}}\right)=-\hat{C}_{x} \sqrt{V_{x}^{2}+V_{y}^{2}+V_{z}^{2}} \cdot \frac{\partial V_{y}}{\partial V_{y 0}} \\
& -\hat{C}_{x}\left(V_{x}^{2}+V_{y}^{2}+V_{z}^{2}\right)^{-\frac{1}{2}} V_{y}^{2} \cdot \frac{\partial V_{y}}{\partial V_{y 0}} \text {, }
\end{aligned}
$$

and

$$
\begin{aligned}
\frac{d}{d t}\left(\frac{\partial V_{z}}{\partial V_{z 0}}\right)= & -\hat{C}_{x} \sqrt{V_{x}^{2}+V_{y}^{2}+V_{z}^{2}} \cdot \frac{\partial V_{z}}{\partial V_{z 0}} \\
& -\hat{C}_{x}\left(V_{x}^{2}+V_{y}^{2}+V_{z}^{2}\right)^{-\frac{1}{2}} V_{z}^{2} \cdot \frac{\partial V_{z}}{\partial V_{z 0}}
\end{aligned}
$$

Additionally, to complete the $\partial \mathbf{V} / \partial \mathbf{V}_{0}$ portion of $\partial \mathbf{X} / \partial \mathbf{X}_{0}$, six off-diagonal terms must be computed for a complete set of derivatives:

$$
\frac{d}{d t}\left(\frac{\partial V_{x}}{\partial V_{y 0}}\right)=-\hat{C}_{x}\left(V_{x}^{2}+V_{y}^{2}+V_{z}^{2}\right)^{-\frac{1}{2}} \cdot V_{y} V_{x} \cdot \frac{\partial V_{y}}{\partial V_{y 0}}
$$




$$
\begin{aligned}
& \frac{d}{d t}\left(\frac{\partial V_{x}}{\partial V_{z 0}}\right)=-\hat{C}_{x}\left(V_{x}^{2}+V_{y}^{2}+V_{z}^{2}\right)^{-\frac{1}{2}} \cdot V_{z} V_{x} \cdot \frac{\partial V_{z}}{\partial V_{z 0}}, \\
& \frac{d}{d t}\left(\frac{\partial V_{y}}{\partial V_{x 0}}\right)=-\hat{C}_{x}\left(V_{x}^{2}+V_{y}^{2}+V_{z}^{2}\right)^{-\frac{1}{2}} \cdot V_{y} V_{x} \cdot \frac{\partial V_{x}}{\partial V_{x 0}}, \\
& \frac{d}{d t}\left(\frac{\partial V_{y}}{\partial V_{z 0}}\right)=-\hat{C}_{x}\left(V_{x}^{2}+V_{y}^{2}+V_{z}^{2}\right)^{-\frac{1}{2}} \cdot V_{y} V_{z} \cdot \frac{\partial V_{z}}{\partial V_{z 0}}, \\
& \frac{d}{d t}\left(\frac{\partial V_{z}}{\partial V_{x 0}}\right)=-\hat{C}_{x}\left(V_{x}^{2}+V_{y}^{2}+V_{z}^{2}\right)^{-\frac{1}{2}} \cdot V_{x} V_{z} \cdot \frac{\partial V_{x}}{\partial V_{x 0}},
\end{aligned}
$$

and

$$
\frac{d}{d t}\left(\frac{\partial V_{z}}{\partial V_{y 0}}\right)=-\hat{C}_{x}\left(V_{x}^{2}+V_{y}^{2}+V_{z}^{2}\right)^{-\frac{1}{2}} \cdot V_{y} V_{z} \cdot \frac{\partial V_{y}}{\partial V_{y 0}}
$$

A Marquardt algorithm (Section 7.3) can then be applied to minimize the prediction error using Eqs. 1a-1f and 3a-3f to forecast model-based measurements, and Eqs.11, 21, and 22 to build the corresponding Jacobian matrix. To emphasize the transient parts of radar elevation and azimuth measurements, only the first $0.1 \mathrm{~s}$ of data is used. After 50 Marquardt iterations, the predicted radar measurements match the recorded data to the degree shown in Fig. 2. The resulting initial condition estimates are shown in Table 1 in the "Estimate (static)" column. Values are reported for $x, y, z$, and $u \approx\|\dot{x} \quad \dot{y} \quad \dot{z}\|_{2}$. 


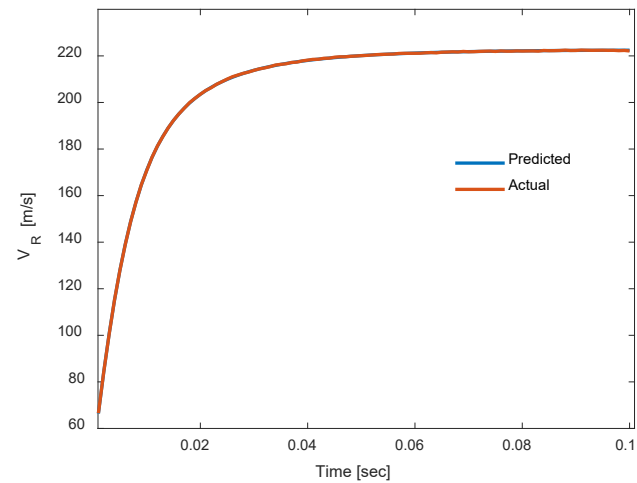

a) Radial Velocity

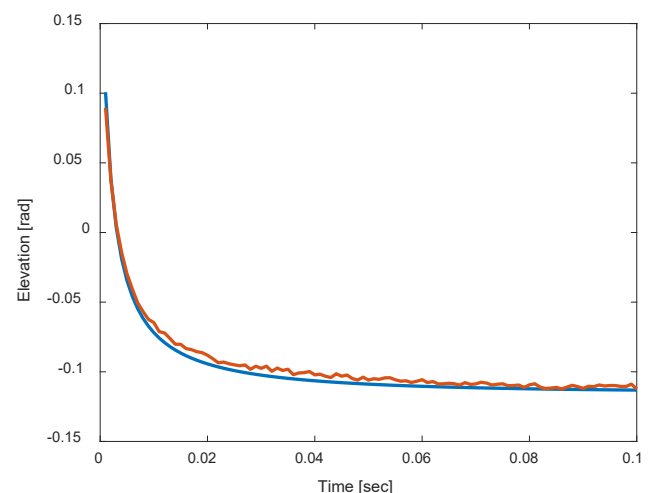

b) Elevation

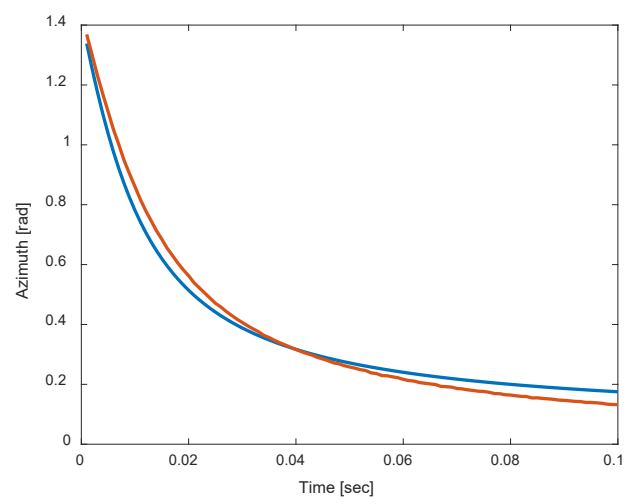

c) Azimuth

Fig. 2 Trajectory matching using a point-mass model with analytic derivatives on the radar data only

\section{Finding Initial Conditions by Merging the UKF with High- Fidelity Dynamics}

A comprehensive set of initial conditions may be found by merging the UKF with a high-fidelity model of the dynamics. Since the UKF uses any set of nonlinear dynamics and nonlinear measurements, existing high-fidelity models may be readily shoe-horned into a UKF algorithm. Figure 3 shows a flow chart of the algorithm using the HMA subroutines authored by Fresconi. ${ }^{6}$ For a more complete review of the UKF, see Burchett. ${ }^{5}$

In order to estimate the initial conditions, the UKF is propagated forward in time until the state estimates are well converged. Typically, the algorithm could then be integrated backward in time as shown in Burchett. ${ }^{5}$ However, for the dual-spin HMA configuration, the tail-kit roll and roll rate are unmeasured and unobservable. This introduces a conundrum where the UKF will converge when integrated forward in time, because the tail-kit states are stable in forward time, and thus the system is detectable. However, since the tail-kit states are unstable in backward 
time, they are both unobservable and undetectable, making the system undetectable. Thus, two additional approaches were contrived to approximate the initial conditions. First, the early history of the plant states could be estimated by backward spline extrapolation from the converged portion of the state estimates. This method rendered adequate estimates as long as the measurements were noise free. However, when more realistic noisy measurements were introduced, the spline method failed. The second method was to start the backward integration at a time briefly after the state estimates had converged and integrate backward without measurement updates. If the states have indeed converged, they should track the true states backward for a short period of time without measurement updates.

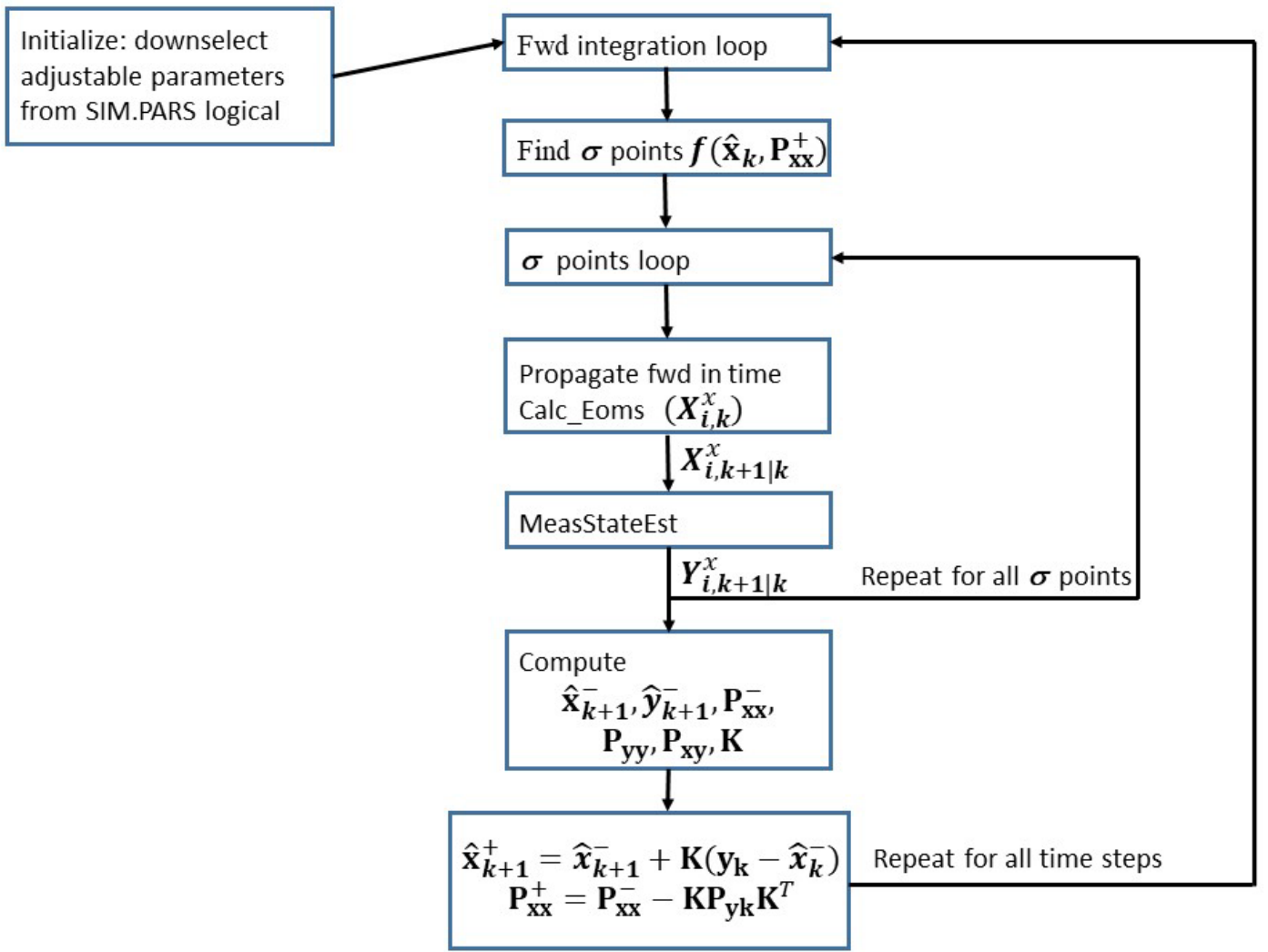

Fig. 3 Flow chart for applying UKF to high-fidelity simulation "HMA"

Figures 4-6 show the state estimates for the second method. Truth values are plotted as green dashed lines for comparison. The forward integration state estimates are plotted as blue lines, and the backward integration predictions are shown as red lines. Forward integration proceeded for the full $0.3 \mathrm{~s}$ of available data. Backward integration was started at $0.1 \mathrm{~s}$ where estimation error transients have largely died out. The Pitch plot is perhaps the best example of this. Note that several state estimates were initialized at zero. Some of the directly measured states such as pitch and yaw rate converge so quickly that the transient-state estimate error 
is indicated by a vertical blue segment at the start of forward integration. Some of the indirectly measured states such as altitude, roll, and yaw never fully converge. Radar azimuth and elevation measures have small sensitivity to changes in cross range and altitude respectively, and forward body-roll rate is plagued by large sensor noise and unmeasured actuator inputs. These effects prevent estimates of the projectile's CG position, roll rate, and roll angle from adequate convergence. 


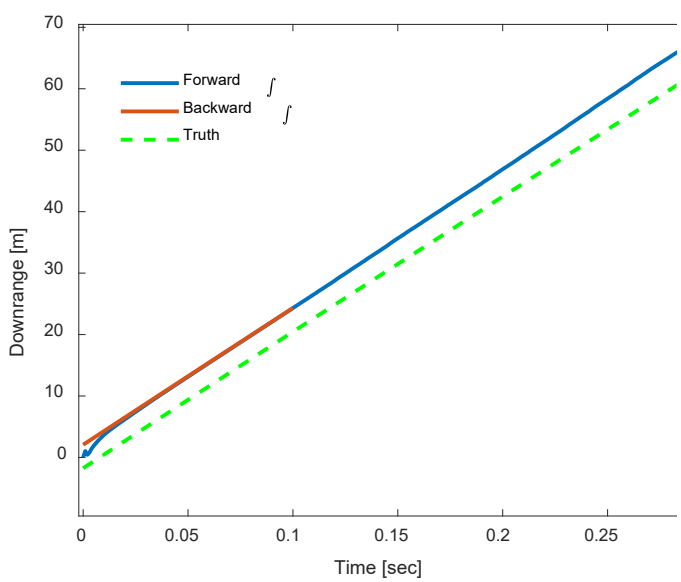

a) Downrange

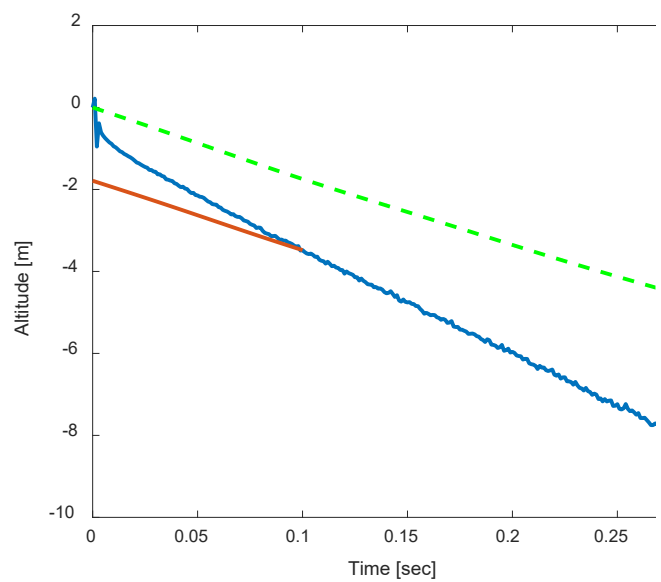

c) Altitude

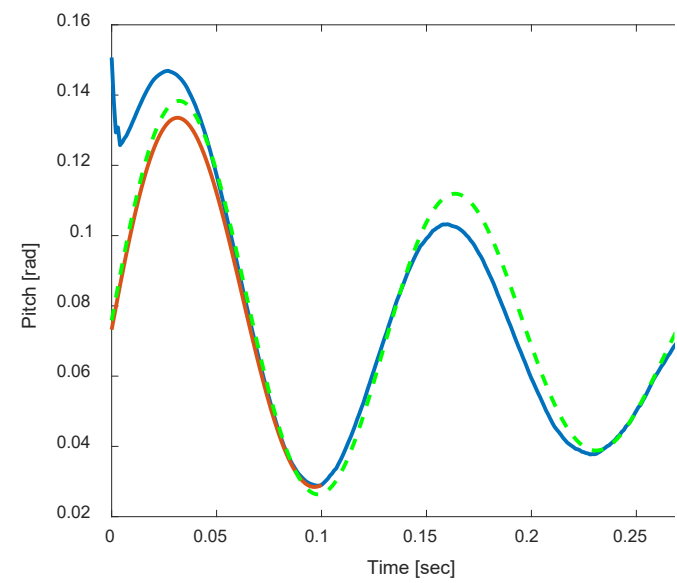

e) Pitch

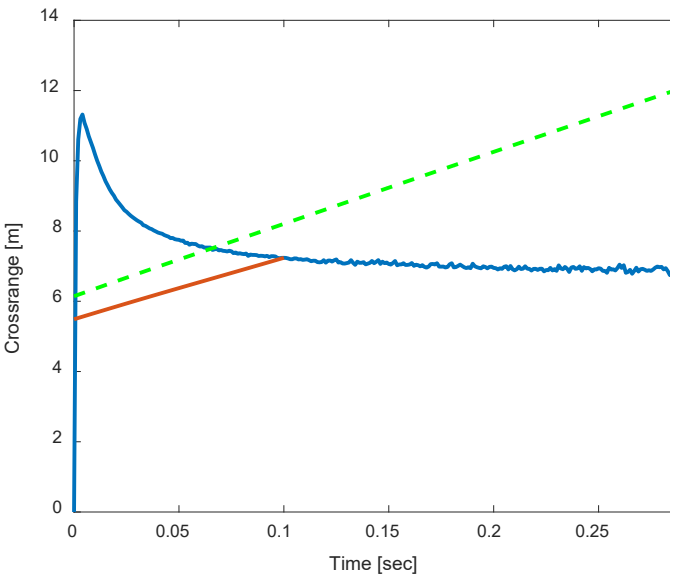

b) Cross range

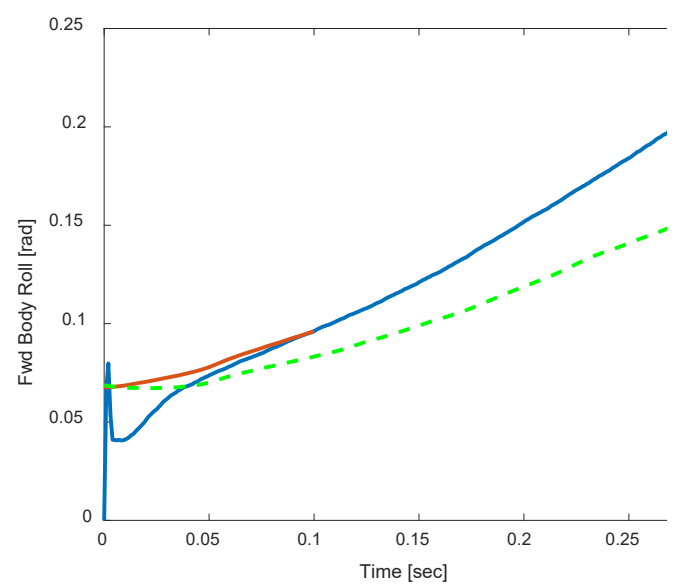

d) Forward body-roll angle

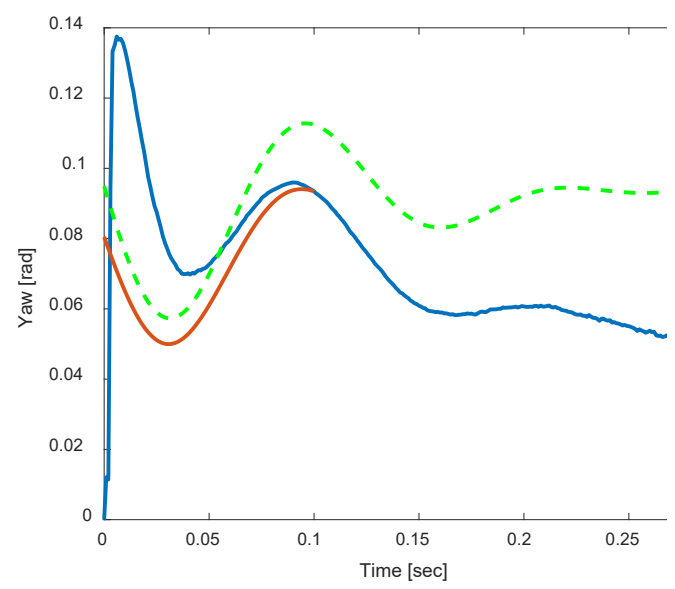

f) Yaw

Fig. 4 Second method's trajectory matching using the UKF with high-fidelity dynamics; backward integration from $0.1 \mathrm{~s}$ ignores the measurements 


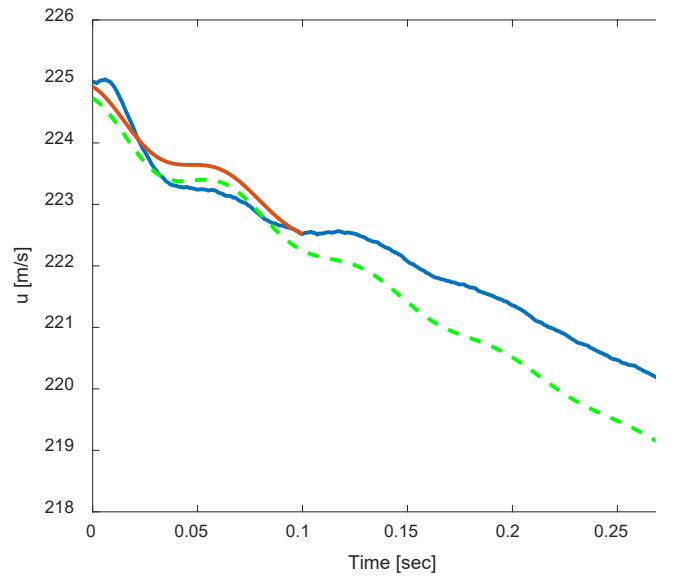

a) Forward velocity

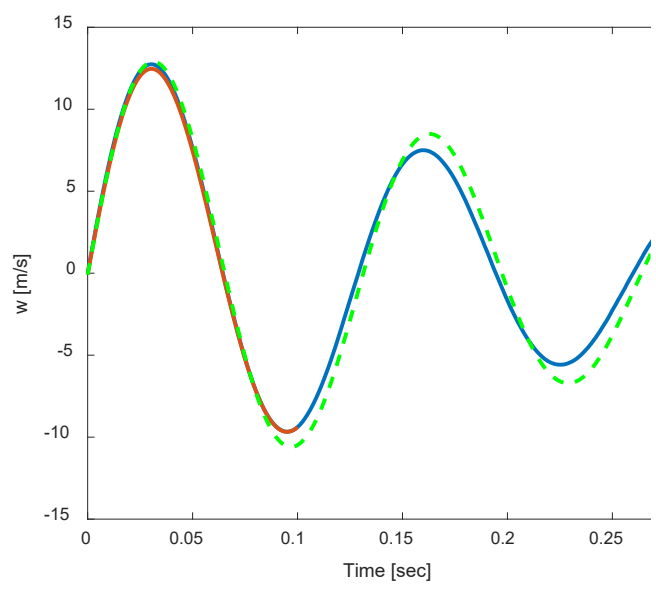

c) Angle of attack velocity

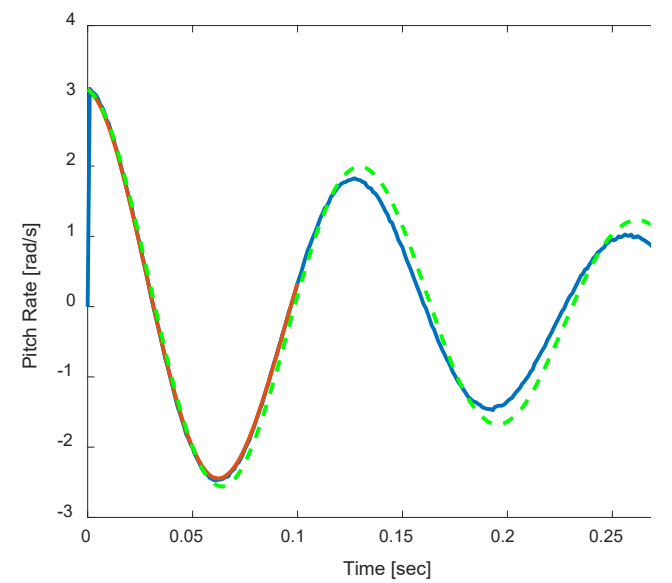

e) Pitch rate

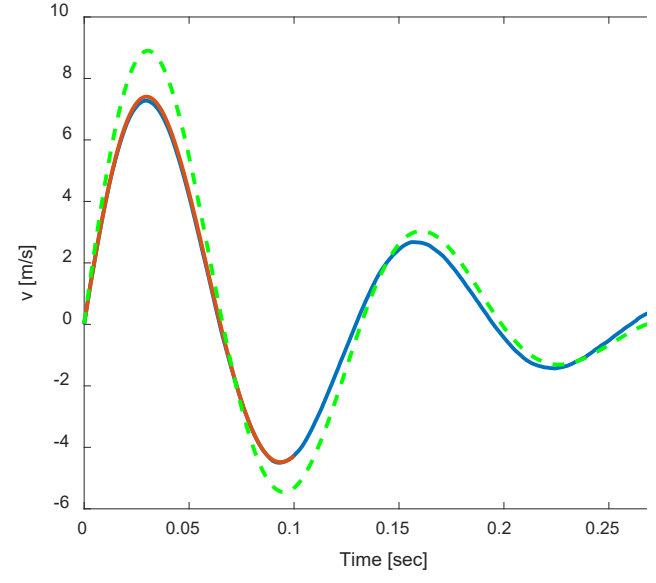

b) Sideslip velocity

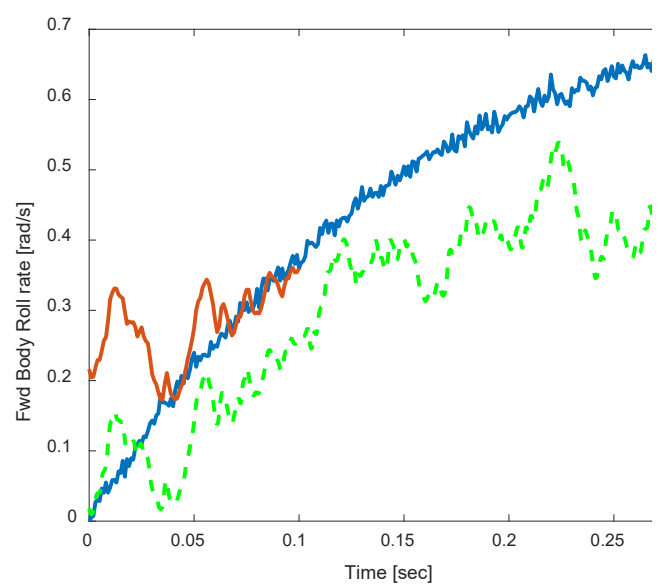

d) Forward body-roll rate

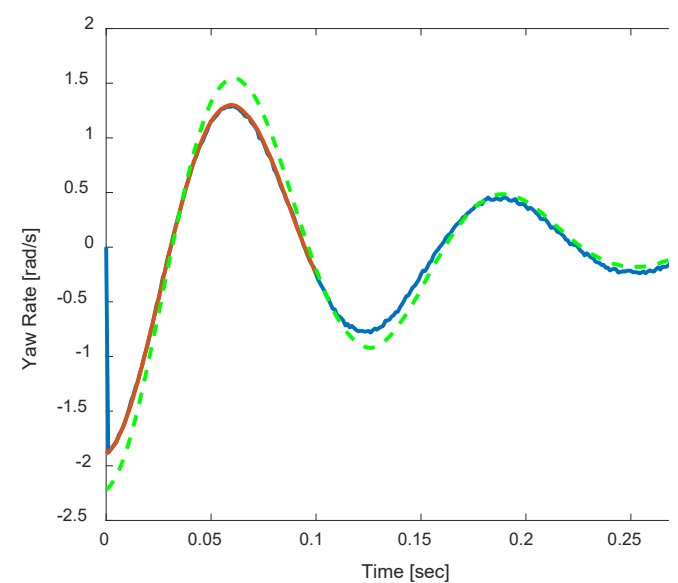

f) Yaw rate

Fig. 5 Trajectory matching in second method using the UKF with high-fidelity dynamics; backward integration from $0.1 \mathrm{~s}$ ignores the measurements 


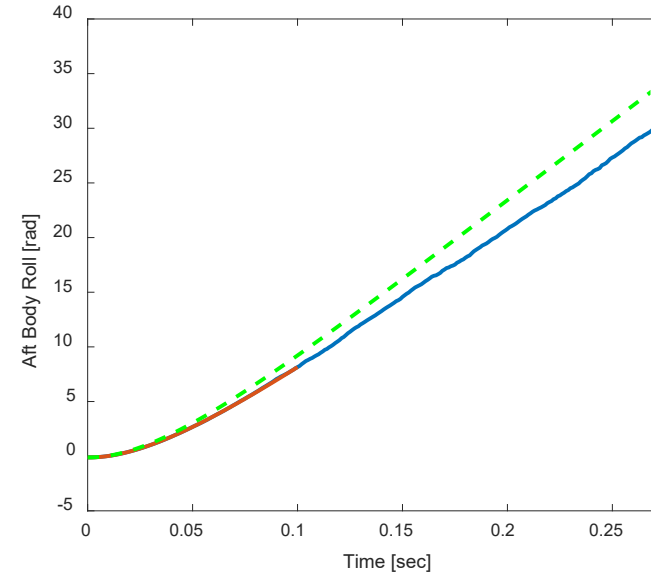

a) Aft body-roll angle

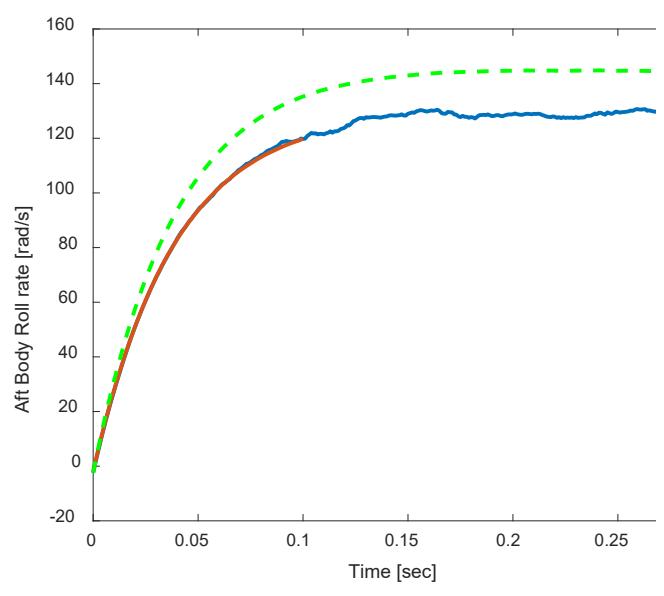

b) Aft body-roll rate

Fig. 6 Trajectory matching using the UKF with high-fidelity dynamics; backward integration from $0.1 \mathrm{~s}$ ignores the measurements

The backward-integration estimates track the truth values well despite the absence of measurement updates. This makes sense since the estimator is using dynamics identical to those of the truth model. Errors at the start of backward integration are not reduced, but they also do not diverge. Thus, good estimates of the initial states are rendered. Table 1 compares the rendered estimates with those from other methods. All with the exception of the unmeasured tail-kit states and $v$ and $w$ are excellent. 
Table 1 Initial-condition estimates rendered by several methods applied to virtual data

\begin{tabular}{ccccc}
\hline State & 7-DOF linear & $\begin{array}{c}\text { UKF of high- } \\
\text { fidelity }\end{array}$ & "Static" models & $\begin{array}{c}\text { NLLS of high- } \\
\text { fidelity }\end{array}$ \\
\hline$x[\mathrm{~m}]$ & $-1.7474^{\mathrm{a}}$ & 2.0693 & -1.7492 & -1.6801 \\
$y[\mathrm{~m}]$ & $6.1567^{\mathrm{a}}$ & 5.4887 & 6.1435 & 6.1707 \\
$z[\mathrm{~m}]$ & $-0.47202^{\mathrm{a}}$ & -1.7877 & -0.45932 & -0.4618 \\
$\phi_{F 0}[\mathrm{rad}]$ & $0.06862^{\mathrm{a}}$ & 0.06756 & 0.068620 & 0.0839 \\
$\theta[\mathrm{rad}]$ & $0.075854^{\mathrm{a}}$ & 0.07322 & 0.0758536 & 0.0673 \\
$\psi[\mathrm{rad}]$ & $0.094949^{\mathrm{a}}$ & 0.080497 & 0.0949494 & 0.0449 \\
$u[\mathrm{~m} / \mathrm{s}]$ & 224.78 & 224.92 & $225.69^{\mathrm{b}}$ & 225.74 \\
$v[\mathrm{~m} / \mathrm{s}]$ & -3.8907 & 0.025264 & $\ldots$ & 0.0477 \\
$w[\mathrm{~m} / \mathrm{s}]$ & -2.7813 & -0.08525 & $\ldots$ & -0.1702 \\
$p_{F 0}[\mathrm{rad} / \mathrm{s}]$ & 0.018171 & 0.21643 & $\ldots$ & 0.3069 \\
$q[\mathrm{rad} / \mathrm{s}]$ & 3.0885 & 3.0964 & $\ldots$ & 3.2567 \\
$r[\mathrm{rad} / \mathrm{s}]$ & -2.2185 & -1.8892 & $\ldots$ & -1.8558 \\
$\phi_{A 0}[\mathrm{rad}]$ & $\ldots$ & -0.11575 & $\ldots$ & -0.1099 \\
$p_{A 0}[\mathrm{rad} / \mathrm{s}]$ & 58.307 & -1.9802 & $\ldots$ & -2.4166 \\
\hline
\end{tabular}

Note: NLLS $=$ nonlinear least squares

a Quantities were seeded from "static" model refinements.

b 2-norm of body-frame velocity

\section{Finding Initial Conditions by Forward Integration of a 7-DOF Linear Model}

Since the backward integration of the high-fidelity dynamics resulted in an undetectable system, we investigated additional methods that used forward integration and gradient-based searches. Parameter updates using batch calculations were preferred over recursive methods such as the UKF. The initial angular rates and linear velocities were found by comparing the onboard sensor measurements with a set of predictions based on a linear 7-DOF model and iterating using the Marquardt algorithm. The gradient information required for Marquardt iteration was found using a technique by Burchett ${ }^{1}$ expanded to the 7-DOF "dualspin" case. $^{2}$

First, we introduce the 7-DOF linear model from Burchett et al. ${ }^{2}$ Assuming flat fire and aerodynamic and mass symmetry and using downrange-distance traveled as the independent variable, a set of linear equations can be used to predict the flight of a dual-spin projectile. Downrange distance is assumed to accumulate at a constant rate, taking the place of time: 


$$
x^{\prime}=D
$$

Flat fire and small angles of attack are assumed such that total velocity is a function of base drag only:

$$
V^{\prime}=-\frac{\rho S D}{2 m} C_{X 0} V
$$

Roll angles are directly integrated from the roll rates:

and

$$
\begin{aligned}
\phi_{F}^{\prime} & =\frac{D}{V} p_{F} \\
\phi_{A}^{\prime} & =\frac{D}{V} P_{A} .
\end{aligned}
$$

The two roll rates are modeled by the following state space and may be coupled by bearing friction, $C_{V}$ :

$$
\left\{\begin{array}{l}
p_{F}{ }^{\prime} \\
p_{A}{ }^{\prime}
\end{array}\right\}=\left[\begin{array}{ll}
H & J \\
T & L
\end{array}\right]\left\{\begin{array}{l}
p_{F} \\
p_{A}
\end{array}\right\}+\left\{\begin{array}{l}
M \\
N
\end{array}\right\}
$$

where

$$
\begin{gathered}
H=\frac{\rho S D^{3}}{4 I_{x x}^{F}} C_{L P}^{F}-J, \\
J=\frac{D C_{V}}{V I_{x x}^{F}}, \\
L=\frac{\rho S D^{3}}{4 I_{x x}^{A}} C_{L P}^{A}-T, \\
T=\frac{D C_{V}}{V I_{x x}^{A}}, \\
M=\frac{\rho S D^{2} V}{2 I_{x x}^{F}} C_{L D D}^{F},
\end{gathered}
$$

and

$$
N=\frac{\rho S D^{2} V}{2 I_{x x}^{A}} C_{L D D}^{A}
$$


The angle of attack, sideslip, pitch rate, and yaw rate are measured in a no-roll frame and may be modeled as the following linear system:

$$
\left\{\begin{array}{c}
v^{\prime} \\
w^{\prime} \\
q^{\prime} \\
r^{\prime}
\end{array}\right\}=\Xi\left\{\begin{array}{c}
v \\
w \\
q \\
r
\end{array}\right\}+\left\{\begin{array}{l}
0 \\
1 \\
0 \\
0
\end{array}\right\} G g
$$

where epicyclic frequencies and mode shapes are governed by the following matrix:

$$
\Xi=\left[\begin{array}{cccc}
-\Xi_{1} & 0 & 0 & -D \\
0 & -\Xi_{1} & D & 0 \\
0 & \Xi_{3} & \Xi_{4} & -\Xi_{5} \\
-\Xi_{3} & 0 & \Xi_{5} & \Xi_{4}
\end{array}\right]
$$

and

$$
\begin{aligned}
& \Xi_{1}=\frac{\rho S D}{2 m} C_{N A}, \\
& \Xi_{3}=\frac{\rho S D^{2}}{2 I_{y y}} C_{M A}, \\
& \Xi_{4}=\frac{\rho S D^{3}}{4 I_{y y}} C_{M Q},
\end{aligned}
$$

and

$$
\Xi_{5}=\frac{D}{V} \frac{\left(I_{X X}^{F} p_{F}+I_{X X}^{A} p_{A}\right)}{I_{y y}}
$$

Cross range, altitude, pitch, and yaw are modeled by the following linear system with epicyclic states treated as a forcing function:

$$
\left\{\begin{array}{c}
y^{\prime} \\
z^{\prime} \\
\theta^{\prime} \\
\psi^{\prime}
\end{array}\right\}=\boldsymbol{\Phi}\left\{\begin{array}{l}
y \\
z \\
\theta \\
\psi
\end{array}\right\}+\frac{D}{V} \mathbf{I}\left\{\begin{array}{c}
v \\
w \\
q \\
r
\end{array}\right\}
$$

and

$$
\boldsymbol{\Phi}=\left[\begin{array}{cccc}
0 & 0 & 0 & D \\
0 & 0 & -D & 0 \\
0 & 0 & 0 & 0 \\
0 & 0 & 0 & 0
\end{array}\right]
$$




\subsection{Solution for the Linear 7-DOF Model}

The model can be analytically solved for its trajectory as follows. Total velocity $(V)$, roll rates, and epicyclic pitch rate, yaw rate, angle of attack, and sideslip are treated as priors that must be solved in that order before integrating for the linear and angular position states. Forward velocity for flat fire is approximated by

$$
V(s)=V_{0} \exp \left(-\frac{\rho S D}{2 m} C_{X 0} s\right)
$$

Equation 27 has the form

$$
\xi^{\prime}(s)=\mathbf{A} \xi(s)+\mathbf{B}(s),
$$

the total solution of which is given as

$$
\xi(s)=e^{\mathbf{A} s} \xi_{0}+e^{\mathbf{A} s} \int_{0}^{s} e^{-\mathbf{A} \tau} \mathbf{B}(\tau) d \tau .
$$

The integration may be performed by a matrix exponential as given in Van Loan. ${ }^{3}$ If, for instance,

then

$$
\boldsymbol{\Psi}=\left[\begin{array}{cc}
\mathbf{A} & \mathbf{B} \\
0 & R
\end{array}\right]
$$

$$
e^{\Psi_{s}}=\left[\begin{array}{cc}
\boldsymbol{\Omega}_{1} & \boldsymbol{\Delta}_{\mathbf{1}} \\
0 & \Omega_{2}
\end{array}\right]
$$

and

$$
\begin{gathered}
\mathbf{\Omega}_{\mathbf{1}}=e^{\mathbf{A} s} \\
\boldsymbol{\Delta}_{\mathbf{1}}=\boldsymbol{\Omega}_{1} \int_{0}^{s} e^{-\mathbf{A} \tau} \mathbf{B}(\tau) d \tau .
\end{gathered}
$$

Thus, if we set

$R=0$, and

$$
\mathbf{A}=\left[\begin{array}{ll}
H & J \\
T & L
\end{array}\right]
$$

$$
\mathbf{B}=\left\{\begin{array}{l}
M \\
N
\end{array}\right\} \text {. }
$$

Using the average total velocity over the previous two samples in $M$ and $N$, we can use Eqs. 34-38 to find the roll rates at the next sample such that

$$
\xi(s+1)=\mathbf{\Omega}_{\mathbf{1}} \xi(s)+\boldsymbol{\Delta}_{\mathbf{1}} .
$$

Eq. 28 can then be assembled using the average roll rates and average total velocity over the last two samples in $\Xi_{5}$. Equation 28 is solved by separating into homogeneous and particular solutions. The particular solution is given by setting the left-hand side to zero and solving for the steady-state values: 


$$
\eta_{p}=\left\{\begin{array}{c}
v_{p} \\
w_{p} \\
q_{p} \\
r_{p}
\end{array}\right\}=-\Xi^{-1}\left\{\begin{array}{l}
0 \\
G \\
0 \\
0
\end{array}\right\} g .
$$

The particular solution is then subtracted from the initial conditions prior to solving for the homogeneous part

$$
\chi=\eta(s)-\eta_{p}
$$

The solution is then given by matrix exponentiation and adding the homogeneous solution back in

$$
\eta(s+1)=e^{\Xi s} \chi+\eta_{p}
$$

where $\eta=\left\{\begin{array}{llll}v & w & q & r\end{array}\right\}^{T}$.

Now since Eq. 30 has the same form as Eq.33, we use Eqs. 34-38 to find the position states at the next sample, making the following substitutions: $\mathbf{A}=\boldsymbol{\Phi}$ and

$$
\mathbf{B}=\frac{D}{V} \mathbf{I}\left\{\begin{array}{c}
v \\
w \\
q \\
r
\end{array}\right\},
$$

where the epicyclic states are averaged over the past two samples.

\subsection{Differentiating the Solution with Respect to the Initial Conditions}

The derivatives of roll rates and position variables can be found by differentiating Eq. 41 with respect to the variables of interest. First, collect the two roll rates into a roll rate vector $\mathbf{P}=\left\{\begin{array}{ll}p_{F} & p_{A}\end{array}\right\}^{T}$. It follows that $\partial \mathbf{P} /\left.\partial \mathbf{P}_{0}\right|_{s=0}=\mathbf{I}$. Since $\mathbf{A}, \mathbf{B}$ and hence $\boldsymbol{\Omega}_{1}, \boldsymbol{\Delta}_{1}$ are invariant wrt $\mathbf{P}_{0}$, Eq. 41 may then be applied recursively to predict the entire sequence of derivatives

$$
\left.\frac{\partial \mathbf{P}}{\partial \mathbf{P}_{0}}\right|_{s+1}=\left.\boldsymbol{\Omega}_{1} \frac{\partial \mathbf{P}}{\partial \mathbf{P}_{0}}\right|_{s}
$$

And, the parameters in $\boldsymbol{\Omega}_{1}$ are updated every sampling period.

Likewise, the epicyclic velocity states will require a matrix of costates that are initialized as $\partial \eta /\left.\partial \eta_{0}\right|_{s=0}=\mathbf{I}$. These are propagated along the trajectory by recursively applying Eq. 44: 


$$
\left.\frac{\partial \eta}{\partial \eta_{0}}\right|_{s+1}=\left.e^{\Xi s} \frac{\partial \eta}{\partial \eta_{0}}\right|_{s}
$$

Additionally, sensitivities of the roll states wrt their initial conditions will influence the $\eta$ vector. These quantities may be found by differentiating Eq. 44, keeping in mind that $\Xi$ is a function of the roll rates through the $F$ coefficient. Thus, for instance,

$$
\left.\frac{\partial \eta}{\partial p_{F 0}}\right|_{s+1}=s \frac{\partial \Xi}{\partial p_{F 0}} e^{\Xi s} \chi+e^{\Xi s} \frac{\partial \chi}{\partial p_{F 0}}+\frac{\partial \eta_{p}}{\partial p_{F 0}}
$$

where

$$
\frac{\partial \Xi}{\partial p_{F 0}}=\left[\begin{array}{cccc}
0 & 0 & 0 & 0 \\
0 & 0 & 0 & 0 \\
0 & 0 & 0 & -\partial \Xi_{5} / \partial p_{F 0} \\
0 & 0 & \partial \Xi_{5} / \partial p_{F 0} & 0
\end{array}\right]
$$

and

$$
\frac{\partial \mathbf{\Xi}_{5}}{\partial p_{F 0}}=\left.\frac{D}{V} \frac{I_{X X}^{F}}{I_{y y}}\left[\begin{array}{ll}
1 & 0
\end{array}\right] \frac{\partial \mathbf{P}}{\partial p_{F 0}}\right|_{s}+\left.\frac{D}{V} \frac{I_{X X}^{A}}{I_{y y}}\left[\begin{array}{ll}
0 & 1
\end{array}\right] \frac{\partial \mathbf{P}}{\partial p_{F 0}}\right|_{s} .
$$

The sensitivities of roll states and epicyclic states wrt their own initial conditions will influence the position states such that Eq. 41 will be invoked several times to propagate costates that contain the sensitivities of $\xi=\left\{\begin{array}{llllll}y & z & \theta & \psi\end{array}\right\}^{T}$ wrt all angular rates plus $v$ and $w$.

For example,

$$
\left.\frac{\partial \xi}{\partial v_{0}}\right|_{s+1}=\left.\boldsymbol{\Omega}_{1} \frac{\partial \xi}{\partial v_{0}}\right|_{s}+\frac{\partial \boldsymbol{\Delta}_{1}}{\partial v_{0}}
$$

where $\boldsymbol{\Omega}_{1}$ and $\partial \boldsymbol{\Delta}_{1} / \partial v_{0}$ are found from the matrix exponentiation

and

$$
\exp \left(s \frac{\partial}{\partial v_{0}} \boldsymbol{\Psi}\right)=\left[\begin{array}{cc}
\boldsymbol{\Omega}_{\mathbf{3}} & \frac{\partial \boldsymbol{\Delta}_{\mathbf{1}}}{\partial v_{0}} \\
0 & \Omega_{4}
\end{array}\right]
$$

$$
\frac{\partial}{\partial v_{0}} \boldsymbol{\Psi}=\left[\begin{array}{cc}
\mathbf{A} & \frac{\partial}{\partial v_{0}} \mathbf{B} \\
0 & 0
\end{array}\right]
$$


And, finally, $\partial \mathbf{B} / \partial v_{0}$ is found from averaging the relevant costates over that past two samples

$$
\frac{\partial}{\partial v_{0}} \mathbf{B}=\frac{D}{2 V}\left(\left.\frac{\partial \eta}{\partial v_{0}}\right|_{s}+\left.\frac{\partial \eta}{\partial v_{0}}\right|_{s+1}\right)
$$

For the HMA dual-spin model, derivatives of the state history wrt the following vector of parameters were propagated along the trajectory:

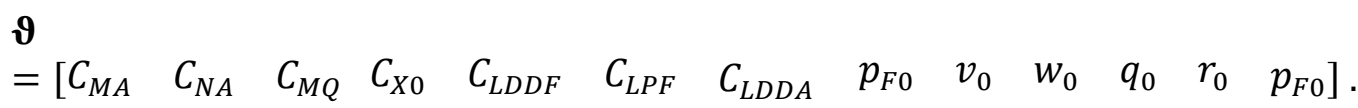

These derivatives are then assembled into a $12 \times 14$ matrix, which represents the derivatives of the state at a given sample wrt the adjustable parameters $\partial \mathbf{X} / \partial \boldsymbol{\vartheta}$.

\subsection{Finding Best Model Parameters through the Marquardt Algorithm}

Onboard sensor measurements were generated using a high-fidelity simulation of the 83-mm HMA vehicle using a sampling rate of $1 \mathrm{kHz}$ and a time horizon of $1 \mathrm{~s}$. These data were then down-sampled onto a grid defined by each full meter of downrange travel. To ease matrix indexing in Matlab, the downrange datum was redefined to be $1 \mathrm{~m}$ downrange. A drag reduction using the method of Section 5 to match radar measurements with a point-mass model was needed in order to provide an estimate of the downrange history corresponding to the collected measurements. With a launch velocity of approximately $225 \mathrm{~m}, 1 \mathrm{~s}$ of travel resulted in 219 samples from $s=1 \mathrm{~m}$ to $s=219 \mathrm{~m}$. The linear model solution and derivatives were used to iterate the model parameters using a Marquardt optimization scheme. The algorithm proceeds as follows:

- $\quad$ Set Marquardt parameters $\mu=0.01$ and $\beta=10$.

- Form the Jacobian matrix and residual vector from data and predictions. Since nine measurements are taken per sample the measurements are interleaved so that all samples of the magnetometer $x$ axis appear on the first 219 rows of $\mathbf{R}$ and $\mathbf{J}$ and so on: 


$$
\mathbf{J}=\left[\begin{array}{ccc}
\mid & \mid & \\
\frac{\partial m_{E x}}{\partial C_{M A}} & \frac{\partial m_{E x}}{\partial C_{N A}} & \ldots \\
\mid & \mid & \\
\mid & \mid & \\
\frac{\partial m_{E y}}{\partial C_{M A}} & \frac{\partial m_{E y}}{\partial C_{M A}} & \ldots \\
\mid & \mid & \ddots
\end{array}\right], \quad \mathbf{R}=\left\{\begin{array}{c}
\mid \\
\widehat{m}_{E x}-m_{E x} \\
\mid \\
\widehat{m}_{E y}-m_{E y} \\
\vdots \\
\vdots
\end{array}\right.
$$

- Make a trial correction

$$
\boldsymbol{\vartheta}^{i+1}=\boldsymbol{\vartheta}^{i}-\left(\mathbf{J}^{T} \mathbf{J}+\mu \mathbf{I}\right)^{-1} \mathbf{J}^{T} \mathbf{R}
$$

- Find the updated residual based by integrating forward in time with parameters $\boldsymbol{\vartheta}^{i+1}$.

- If the error $\|\mathbf{R}\|_{2}$ is reduced, set $\mu=\mu / \beta$ and repeat from Step 2. If not, set $\mu=\mu \beta$ and repeat from Step 3 .

Since only the initial angular rates, angle of attack, and sideslip values are iterated in this context, the remaining initial states were estimated through other methods described in this report. The algorithm is then able to match the actual measurements with model predictions as shown in Fig. 7. 


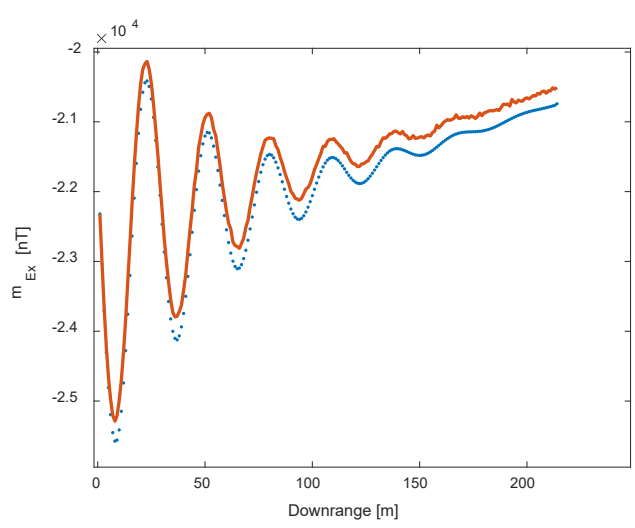

a) Magnetometer $\boldsymbol{x}$ axis

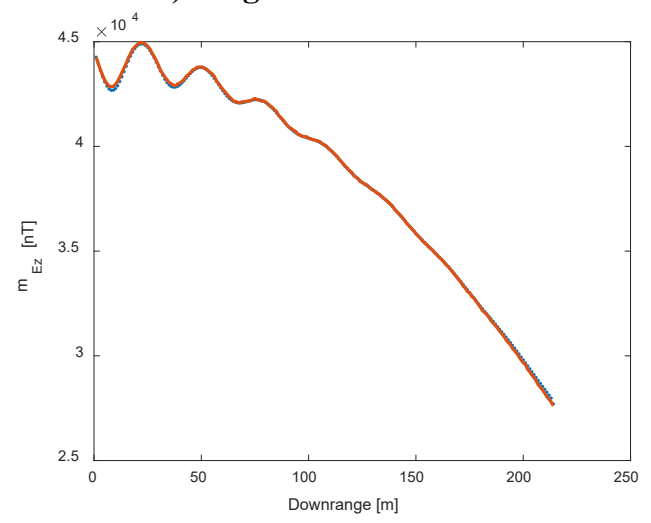

c) Magnetometer $z$ axis

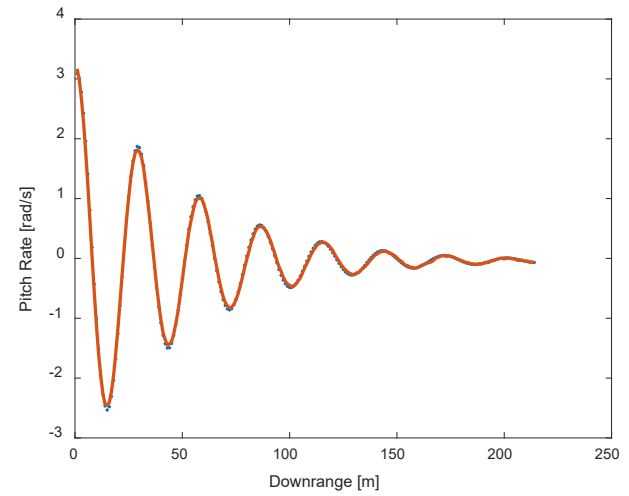

e) Pitch Rate

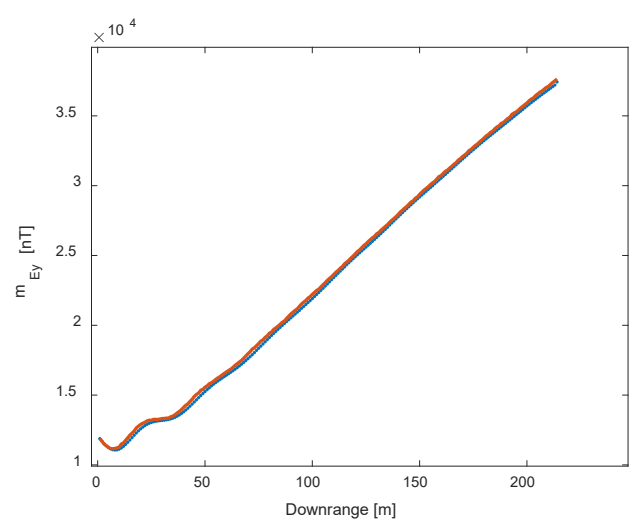

b) Magnetometer $y$ axis

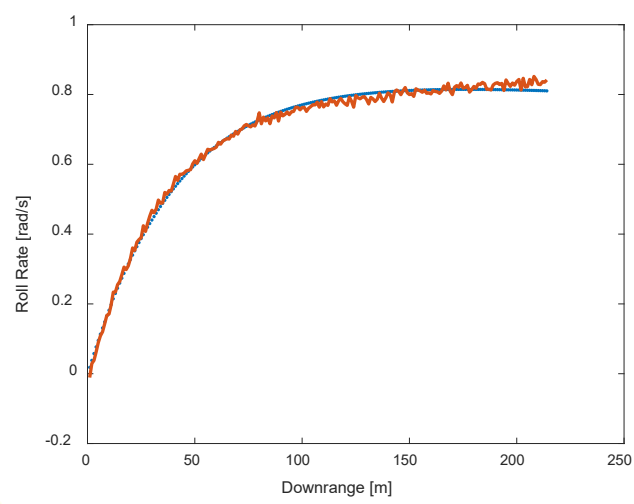

d) Roll Rate

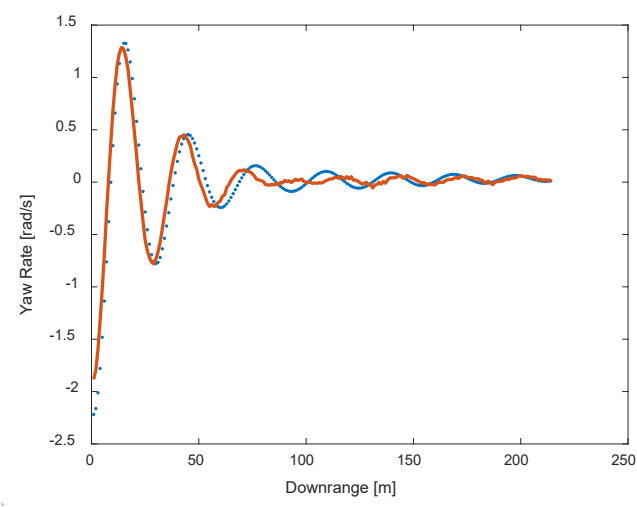

f) Yaw Rate

Fig. 7 Trajectory matching using the 7-DOF linear model with Marquardt algorithm 


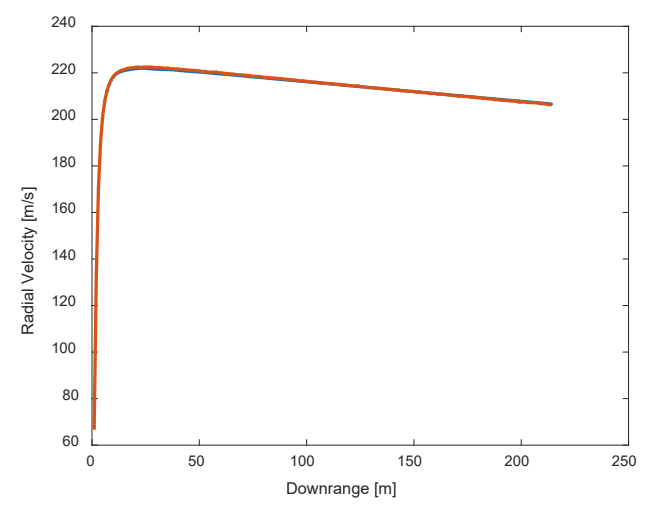

g) Radar Radial Velocity

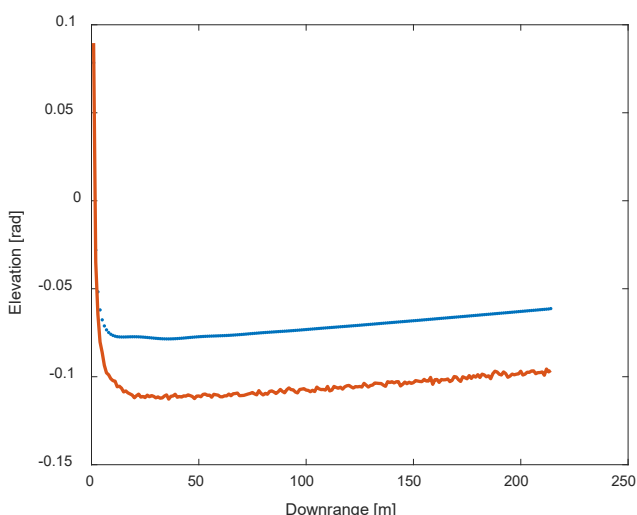

h) Radar Elevation

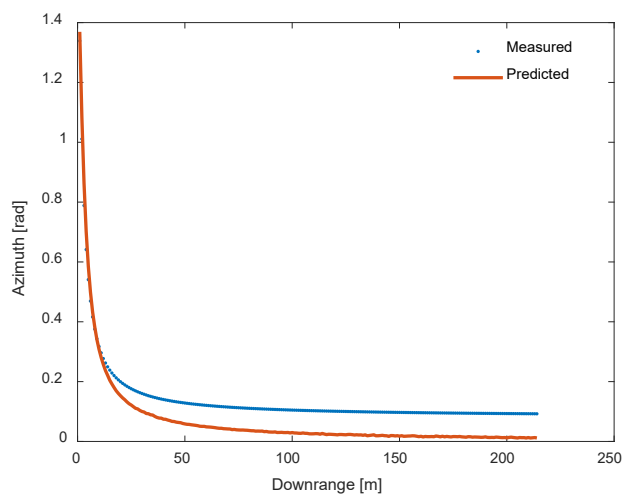

i) Radar Azimuth

Fig. 7 Trajectory matching using the 7-DOF linear model with Marquardt algorithm (continued)

\section{Sensitivities of a Nonlinear 6-DOF Model wrt Initial Conditions}

In order to estimate all 12 initial states in parallel with NLLS estimation of aerodynamic parameters, analytic derivatives of states wrt initial conditions are found. Starting with the standard nonlinear 6-DOF equations of motion (Eqs.2a$2 \mathrm{~d}),{ }^{4}$ derivatives are found in the same fashion as for the 7-DOF linear model.

The far RHS of Eqs. 2a-2d is intended to show the "superficial" sets of independent variables in each expressions. "Superficial" indicates that functional dependencies considered are only those that are explicitly shown in Eqs.2a-2d. Equation 2c is a function of the Euler angles due to the gravity component of the total force vector $\left\{\begin{array}{lll}X & Y & Z\end{array}\right\}^{T}$. Other independent variables are embedded in the aerodynamic force and moment models as will be investigated later. Once again, the chain rule is invoked as 


$$
\frac{d}{d t}\left(\frac{\partial \mathbf{X}}{\partial \mathbf{X}_{0}}\right)=\frac{\partial \dot{\mathbf{X}}}{\partial \mathbf{X}} \frac{\partial \mathbf{X}}{\partial \mathbf{X}_{0}},
$$

resulting in the following non-zero derivatives

$$
\begin{aligned}
& \frac{\partial}{\partial \phi_{0}}\left\{\begin{array}{c}
\dot{x} \\
\dot{y} \\
\dot{z}
\end{array}\right\}=\frac{\partial T_{b 2 i}}{\partial \phi}\left\{\begin{array}{l}
u \\
v \\
w
\end{array}\right\} \frac{\partial \phi}{\partial \phi_{0}}, \\
& \frac{\partial}{\partial \theta_{0}}\left\{\begin{array}{c}
\dot{x} \\
\dot{y} \\
\dot{z}
\end{array}\right\}=\frac{\partial T_{b 2 i}}{\partial \theta}\left\{\begin{array}{l}
u \\
v \\
w
\end{array}\right\} \frac{\partial \theta}{\partial \theta_{0}}, \\
& \frac{\partial}{\partial \psi_{0}}\left\{\begin{array}{c}
\dot{x} \\
\dot{y} \\
\dot{z}
\end{array}\right\}=\frac{\partial T_{b 2 i}}{\partial \psi}\left\{\begin{array}{c}
u \\
v \\
w
\end{array}\right\} \frac{\partial \psi}{\partial \psi_{0}}, \\
& \frac{\partial}{\partial u_{0}}\left\{\begin{array}{c}
\dot{x} \\
\dot{y} \\
\dot{z}
\end{array}\right\}=T_{b 2 i}\left\{\begin{array}{l}
1 \\
0 \\
0
\end{array}\right\} \frac{\partial u}{\partial u_{0}}, \\
& \frac{\partial}{\partial v_{0}}\left\{\begin{array}{c}
\dot{x} \\
\dot{y} \\
\dot{z}
\end{array}\right\}=T_{b 2 i}\left\{\begin{array}{l}
0 \\
1 \\
0
\end{array}\right\} \frac{\partial v}{\partial v_{0}}, \\
& \frac{\partial}{\partial w_{0}}\left\{\begin{array}{c}
\dot{x} \\
\dot{y} \\
\dot{z}
\end{array}\right\}=T_{b 2 i}\left\{\begin{array}{l}
0 \\
0 \\
1
\end{array}\right\} \frac{\partial w}{\partial w_{0}}, \\
& \frac{\partial}{\partial \phi_{0}}\left\{\begin{array}{c}
\dot{\phi} \\
\dot{\theta} \\
\dot{\psi}
\end{array}\right\}=\frac{\partial T_{b 2 e}}{\partial \phi}\left\{\begin{array}{l}
p \\
q \\
r
\end{array}\right\} \frac{\partial \phi}{\partial \phi_{0}}, \\
& \frac{\partial}{\partial \theta_{0}}\left\{\begin{array}{c}
\dot{\phi} \\
\dot{\theta} \\
\dot{\psi}
\end{array}\right\}=\frac{\partial T_{b 2 e}}{\partial \theta}\left\{\begin{array}{l}
p \\
q \\
r
\end{array}\right\} \frac{\partial \theta}{\partial \theta_{0}},
\end{aligned}
$$

and $\partial \psi /\left(\partial \psi_{0}\right)=1$. Also 


$$
\begin{aligned}
& \frac{\partial}{\partial p_{0}}\left\{\begin{array}{c}
\dot{\phi} \\
\dot{\theta} \\
\dot{\psi}
\end{array}\right\}=T_{b 2 e}\left\{\begin{array}{l}
1 \\
0 \\
0
\end{array}\right\} \frac{\partial p}{\partial p_{0}}, \\
& \frac{\partial}{\partial q_{0}}\left\{\begin{array}{c}
\dot{\phi} \\
\dot{\theta} \\
\dot{\psi}
\end{array}\right\}=T_{b 2 e}\left\{\begin{array}{l}
0 \\
1 \\
0
\end{array}\right\} \frac{\partial q}{\partial q_{0}}, \\
& \frac{\partial}{\partial r_{0}}\left\{\begin{array}{c}
\dot{\phi} \\
\dot{\theta} \\
\dot{\psi}
\end{array}\right\}=T_{b 2 e}\left\{\begin{array}{l}
0 \\
0 \\
1
\end{array}\right\} \frac{\partial r}{\partial r_{0}}, \\
& \frac{\partial}{\partial \phi_{0}}\left\{\begin{array}{c}
\dot{u} \\
\dot{v} \\
\dot{w}
\end{array}\right\}=\frac{\partial T_{i 2 b}}{\partial \phi}\left\{\begin{array}{l}
0 \\
0 \\
g
\end{array}\right\} \frac{\partial \phi}{\partial \phi_{0}}, \\
& \frac{\partial}{\partial \theta_{0}}\left\{\begin{array}{l}
\dot{u} \\
\dot{v} \\
\dot{w}
\end{array}\right\}=\frac{\partial T_{i 2 b}}{\partial \theta}\left\{\begin{array}{l}
0 \\
0 \\
g
\end{array}\right\} \frac{\partial \theta}{\partial \theta_{0}}, \\
& \frac{\partial}{\partial \psi_{0}}\left\{\begin{array}{c}
\dot{u} \\
\dot{v} \\
\dot{w}
\end{array}\right\}=\frac{\partial T_{i 2 b}}{\partial \psi}\left\{\begin{array}{l}
0 \\
0 \\
g
\end{array}\right\} \frac{\partial \psi}{\partial \psi_{0}}, \\
& \frac{\partial}{\partial u_{0}}\left\{\begin{array}{c}
\dot{u} \\
\dot{v} \\
\dot{w}
\end{array}\right\}=-\mathbf{S}\left\{\begin{array}{l}
1 \\
0 \\
0
\end{array}\right\} \frac{\partial u}{\partial u_{0}}, \\
& \frac{\partial}{\partial v_{0}}\left\{\begin{array}{c}
\dot{u} \\
\dot{v} \\
\dot{w}
\end{array}\right\}=-\mathbf{S}\left\{\begin{array}{l}
0 \\
1 \\
0
\end{array}\right\} \frac{\partial v}{\partial v_{0}},
\end{aligned}
$$

and

$$
\frac{\partial}{\partial w_{0}}\left\{\begin{array}{c}
\dot{u} \\
\dot{v} \\
\dot{w}
\end{array}\right\}=-\mathbf{S}\left\{\begin{array}{l}
0 \\
0 \\
1
\end{array}\right\} \frac{\partial w}{\partial w_{0}},
$$




$$
\begin{aligned}
& \frac{\partial}{\partial p_{0}}\left\{\begin{array}{c}
\dot{u} \\
\dot{v} \\
\dot{w}
\end{array}\right\}=-\frac{\partial \mathbf{S}}{\partial p}\left\{\begin{array}{c}
u \\
v \\
w
\end{array}\right\} \frac{\partial p}{\partial p_{0}} \\
& \frac{\partial}{\partial q_{0}}\left\{\begin{array}{c}
\dot{u} \\
\dot{v} \\
\dot{w}
\end{array}\right\}=-\frac{\partial \mathbf{S}}{\partial q}\left\{\begin{array}{c}
u \\
v \\
w
\end{array}\right\} \frac{\partial q}{\partial q_{0}} \\
& \frac{\partial}{\partial r_{0}}\left\{\begin{array}{c}
\dot{u} \\
\dot{v} \\
\dot{w}
\end{array}\right\}=-\frac{\partial \mathbf{S}}{\partial r}\left\{\begin{array}{c}
u \\
v \\
w
\end{array}\right\} \frac{\partial r}{\partial r_{0}} \\
& \frac{\partial}{\partial p_{0}}\left\{\begin{array}{l}
\dot{p} \\
\dot{q} \\
\dot{r}
\end{array}\right\}=-\mathbf{I}^{-1}\left\{\left[\frac{\partial \mathbf{S}}{\partial p}\right][\mathbf{I}]\left\{\begin{array}{l}
p \\
q \\
r
\end{array}\right\}+\mathbf{S}[\mathbf{I}]\left\{\begin{array}{l}
1 \\
0 \\
0
\end{array}\right)\right\} \frac{\partial p}{\partial p_{0}} \\
& \frac{\partial}{\partial q_{0}}\left\{\begin{array}{l}
\dot{p} \\
\dot{q} \\
\dot{r}
\end{array}\right\}=-\mathbf{I}^{-1}\left\{\left[\frac{\partial \mathbf{S}}{\partial q}\right][\mathbf{I}]\left\{\begin{array}{l}
p \\
q \\
r
\end{array}\right\}+\mathbf{S}[\mathbf{I}]\left\{\begin{array}{l}
0 \\
1 \\
0
\end{array}\right\}\right) \frac{\partial q}{\partial q_{0}} \\
& \frac{\partial}{\partial r_{0}}\left\{\begin{array}{c}
\dot{p} \\
\dot{q} \\
\dot{r}
\end{array}\right\}=-\mathbf{I}^{-1}\left\{\left[\frac{\partial \mathbf{S}}{\partial r}\right][\mathbf{I}]\left\{\begin{array}{l}
p \\
q \\
r
\end{array}\right\}+\mathbf{S}[\mathbf{I}]\left\{\begin{array}{l}
0 \\
0 \\
1
\end{array}\right\}\right) \frac{\partial r}{\partial r_{0}}
\end{aligned}
$$

and the necessary matrix derivatives are easily found with the exception of

$$
\begin{gathered}
\frac{\partial T_{b 2 e}}{\partial \phi}=\left[\begin{array}{ccc}
0 & c_{\phi} t_{\theta} & -s_{\phi} t_{\theta} \\
0 & -s_{\phi} & -c_{\phi} \\
0 & c_{\phi} / c_{\theta} & -s_{\phi} / c_{\theta}
\end{array}\right] \\
\frac{\partial T_{b 2 e}}{\partial \theta}=\left[\begin{array}{ccc}
0 & s_{\phi} \sec ^{2} \theta & c_{\phi} \sec ^{2} \theta \\
0 & 0 & 0 \\
0 & s_{\phi} t_{\theta} / c_{\theta} & c_{\phi} t_{\theta} / c_{\theta}
\end{array}\right] .
\end{gathered}
$$


After integration, the terms in Eqs. 55-57 can be assembled into matrix form as

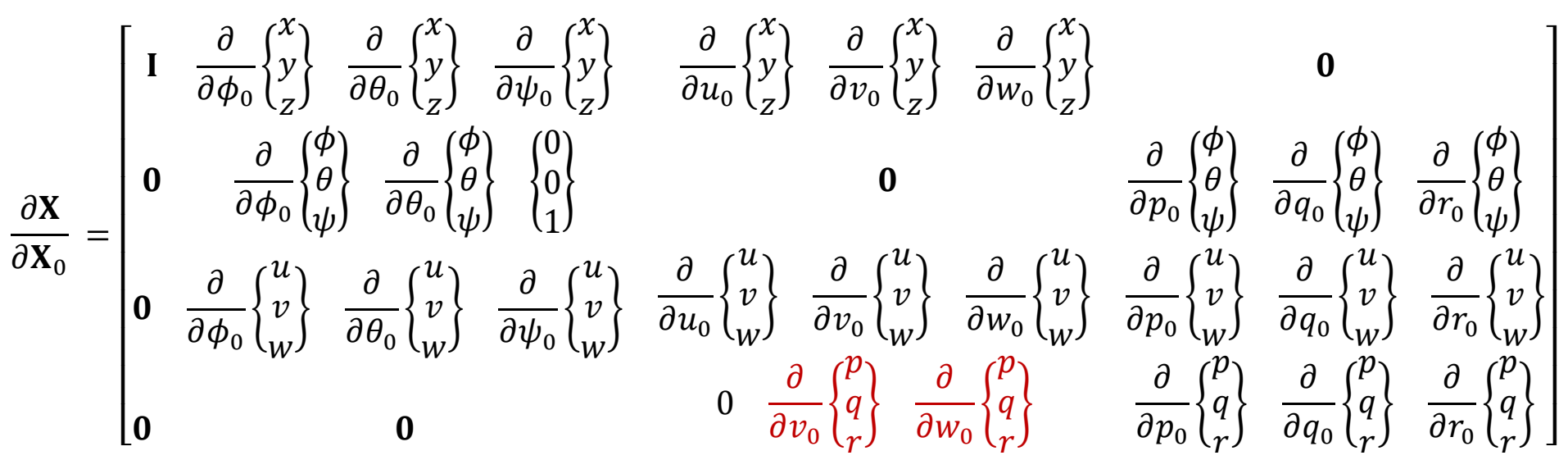


However, the terms highlighted in red are zero under the current assumptions. This is not realistic in light of Eq. 28, where $q$ and $r$ are clearly functions of $v$ and $w$ even in the linear model. In order to precisely model these terms, examine the nonlinear aerodynamic forces and moments. In particular, $q=f(w)$ and $r=g(v)$ due to the moment created by noncollocation of the projectile center of mass and center of pressure. In other words, this coupling stems from the moment found by a cross product of the vector from the CG to the center of pressure with the steady aerodynamic force vector. Assuming that the projectile is axisymmetric in mass and aerodynamics, this becomes

$$
\left\{\begin{array}{l}
L_{S A} \\
M_{S A} \\
N_{S A}
\end{array}\right\}=\left[\begin{array}{ccc}
0 & 0 & 0 \\
0 & 0 & S L_{C G}-S L_{C O P} \\
0 & S L_{C O P}-S L_{C G} & 0
\end{array}\right]\left\{\begin{array}{l}
X_{S A} \\
Y_{S A} \\
Z_{S A}
\end{array}\right\}
$$

Substituting the dynamic pressure, projectile cross sectional area, and angle of attack / sideslip, we can write

$$
\begin{gathered}
M_{S A}=\left(\frac{\pi}{8} \rho V^{2} D^{2}\right)\left(C_{N A} w / V\right)\left(S L_{C G}-S L_{C O P}\right) \\
N_{S A}=-\left(\frac{\pi}{8} \rho V^{2} D^{2}\right)\left(C_{N A} v / V\right)\left(S L_{C G}-S L_{C O P}\right)
\end{gathered}
$$

In the HMA code, the product $C_{N A}\left(S L_{C G}-S L_{C O P}\right)$ is captured in a separate aerodynamic coefficient, $C_{M A}$; however, for consistent units the correct substitution is $C_{N A}\left(S L_{C G}-S L_{C O P}\right) \rightarrow C_{M A} D$. Again assuming mass symmetry, and taking derivatives wrt $w$ and $v$ respectively, the result is

$$
\begin{gathered}
\frac{\partial \dot{q}}{\partial w}=\left(\frac{\pi}{8 I_{y y}} \rho V D^{3}\right) C_{M A} \\
\frac{\partial \dot{r}}{\partial v}=-\left(\frac{\pi}{8 I_{y y}} \rho V D^{3}\right) C_{M A}
\end{gathered}
$$

Thus

$$
\frac{\partial \dot{q}}{\partial w_{0}}=\left(\frac{\pi}{8 I_{y y}} \rho V D^{3}\right) C_{M A} \frac{\partial w}{\partial w_{0}}
$$

and 


$$
\frac{\partial \dot{r}}{\partial v_{0}}=-\left(\frac{\pi}{8 I_{y y}} \rho V D^{3}\right) C_{M A} \frac{\partial v}{\partial v_{0}}
$$

\subsection{Results with Virtual Data}

Matlab modules were written to compute columns of the Jacobian matrix accounting for the sensitivity of onboard sensor measurements with respect to initial conditions using Eqs. 55-58 to compute the time derivatives of the costates needed to populate the matrix $\partial \mathbf{X} / \partial \mathbf{X}_{\mathbf{0}}$. Equations $8,11-16,18$, and 20 are used to evaluate the matrix $\partial \mathbf{Y} / \partial \mathbf{X}$.

These sensitivities were used in the NLLS iteration code provided by Fresconi, which simultaneously searched for four linear aerodynamic coefficients. The search was seeded with initial guesses from the 7-DOF linear approximation with the exceptions of $v$ and $w$. The existing code provided logical arrays to allow the user to down-select a subset of initial conditions to be refined and a subset of measurements to be matched. For program simplicity, the new code does not allow the user to deselect any initial conditions. An additional factor is introduced instead, allowing the user to artificially increase the sensitivities of each column of $\partial \mathbf{X} / \partial \mathbf{X}_{\mathbf{0}}$ and thus slow down the adjustment of selected initial conditions (ICs).

Figure 6 compares the model predictions with the actual sensor data after 15 NLLS iterations. Only the first $0.3 \mathrm{~s}$ of data were used to reduce processing time and emphasize early transients, especially in radar signals. After 15 iterations, initial condition estimates move to the values shown in the far-right column of Table 1. As seen in Fig. 8, model predictions match the sensor signals very well with only slight discrepancies in angular rates and radar azimuth early in the trajectory. 


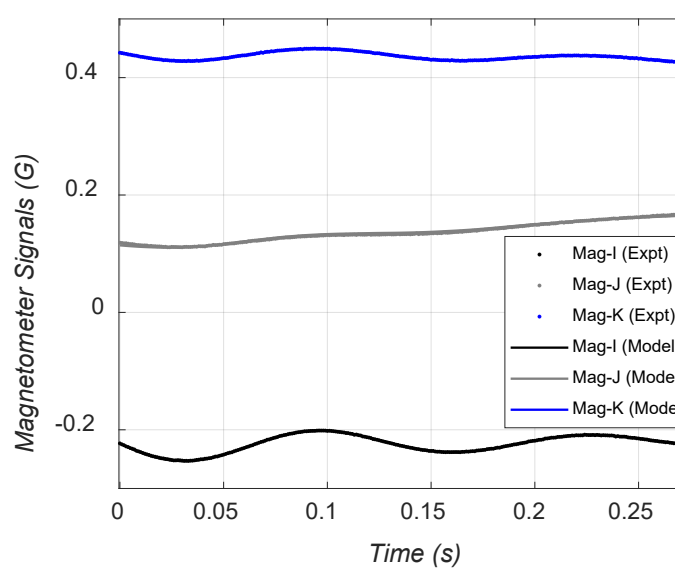

a) Magentometer

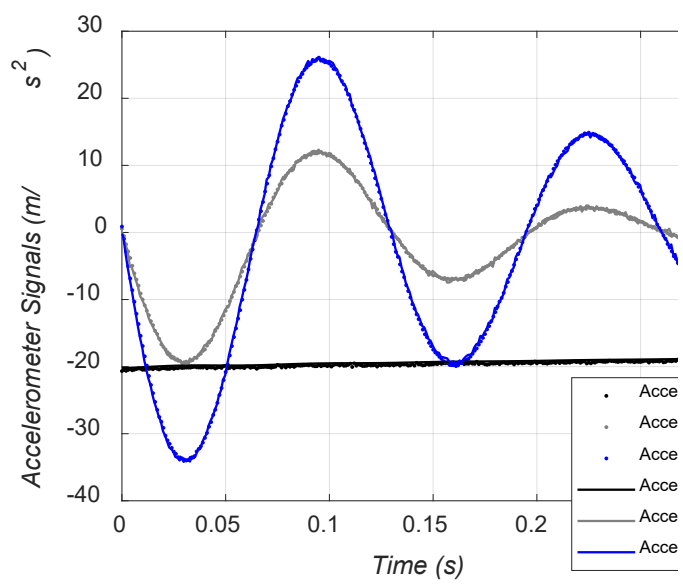

c) Accelerometer

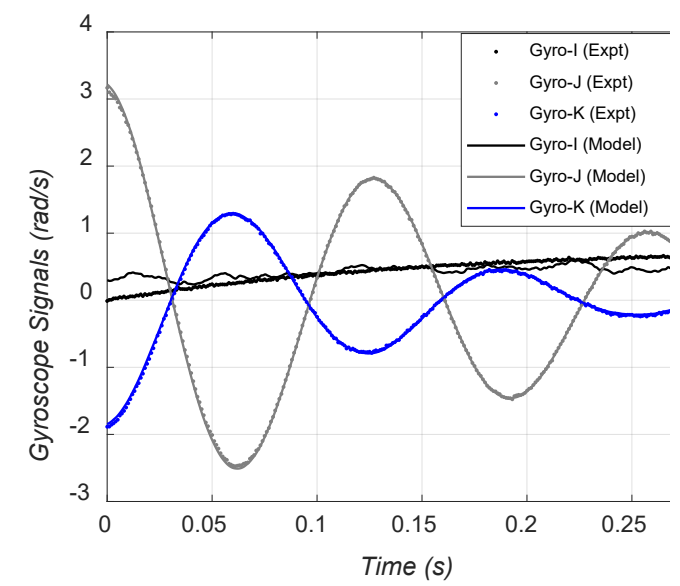

b) Gyroscope

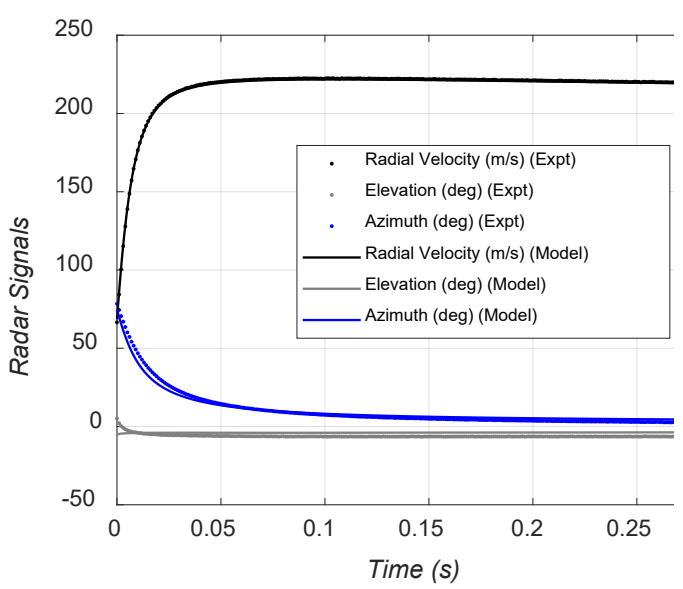

d) Radar

Fig. 8 High-fidelity model predictions of the onboard sensor signals after 15 iterations of NLLS refinements to ICs and linear aerodynamic parameters

\subsection{Results with Actual Data}

Data were collected from the launch of an instrumented 83-mm HMA mortar round. The static algorithms of Section 5 were performed to provide good starting guesses for the Euler angles and position. Once good Euler angles and position were established, the algorithm of this section was applied for 15 iterations. Figure 9 shows the model predictions compared with the actual measurements after modifying all 12 initial conditions and five aerodynamic parameters for 15 iterations. 


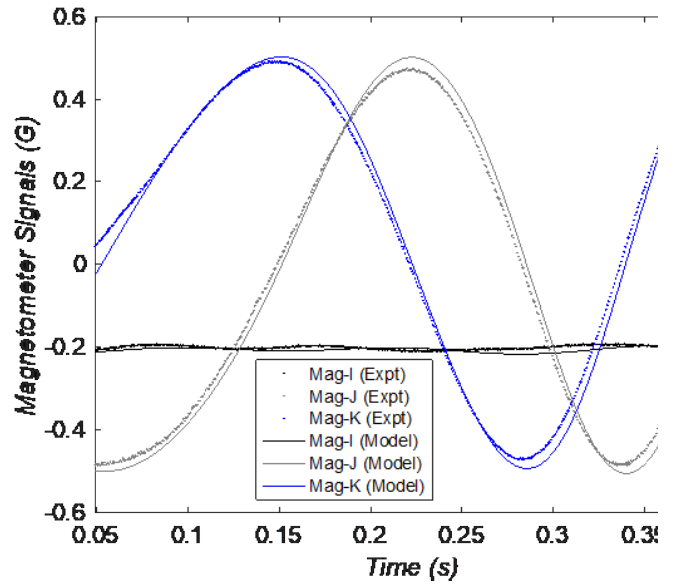

a) Magentometer

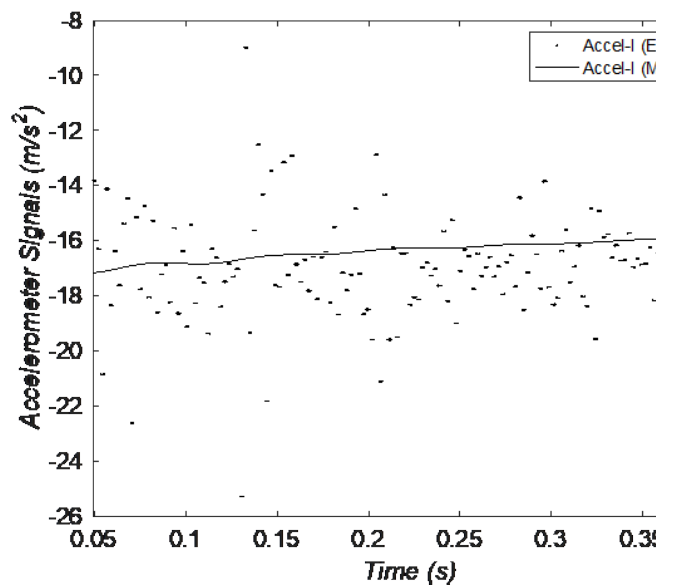

c) Accelerometer

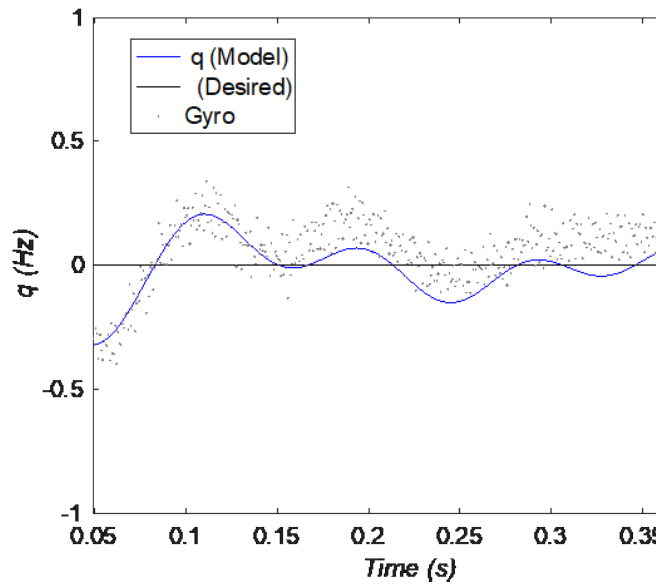

e) Pitch Rate

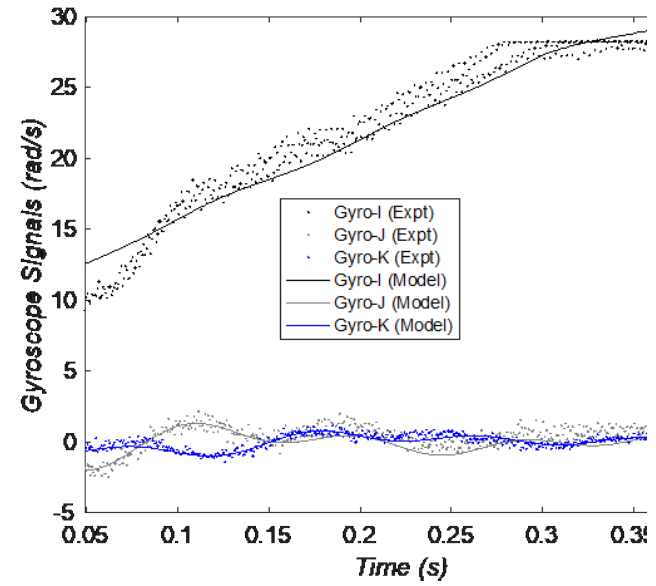

b) Gyroscope

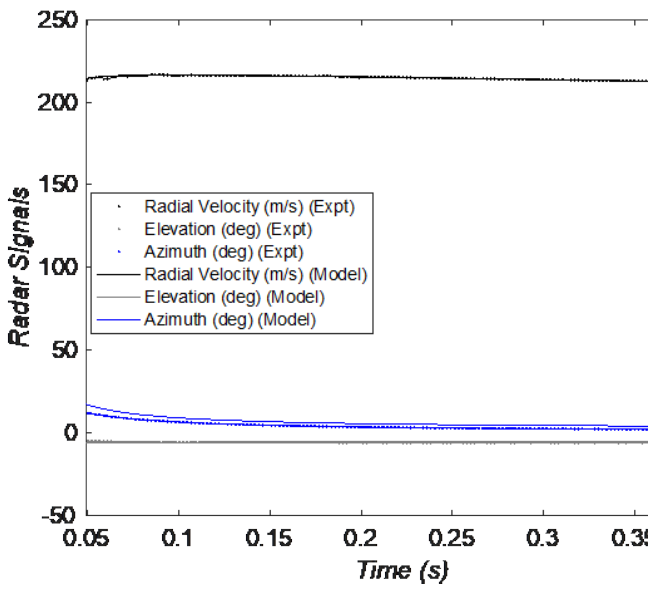

d\} Radar

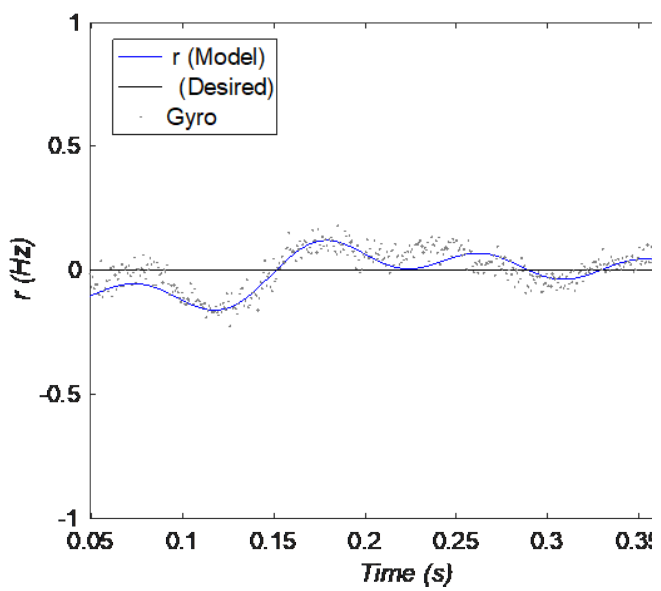

f) Yaw Rate

Fig. 9 Model predictions and actual measurements for HMA Flight 8 data

Note that magnetometer predictions match the data well. The I-axis gyroscope saturates around $28.3 \mathrm{rad} / \mathrm{s}$, making it impossible to observe the actual spin rate beyond about $t=0.28 \mathrm{~s}$. Bias and misalignment errors were estimated for the $\mathrm{J}$ - and 
$\mathrm{K}$-axis gyroscopes assuming a common instrument axis frame. Also, data prior to $49 \mathrm{~ms}$ were ignored due to dead band behavior of certain signals prior to this point. Accelerometer transverse channels were ignored due to large noise. The axial acceleration is plotted and exhibits a similar degree of noise. Since axial acceleration is not modulated by epicyclic pitch and yaw, the model is able to capture the average axial acceleration due primarily to drag. Radar signals were matched well by the model.

Table 2 displays the guesses rendered by static methods and initial and final estimates used in the high-fidelity search. Note that only a 6-DOF model was used, which proved adequate since high-speed camera footage indicated the front and aft sections of the projectile rolled in sync for a large part of the trajectory.

Table 3 shows the aerodynamic parameters that were iterated during the IC search.

Table 2 IC estimates from reducing actual HMA data

\begin{tabular}{cccc}
\hline State & “Static" models & $\begin{array}{c}\text { Guesses entered } \\
\text { into NLLS }\end{array}$ & $\begin{array}{c}\text { Result of NLLS } \\
\text { after 24 iterations }\end{array}$ \\
\hline$x[\mathrm{~m}]$ & 5.0472 & 5.0472 & 8.1277 \\
$y[\mathrm{~m}]$ & -1.8633 & -1.8633 & -0.3235 \\
$z[\mathrm{~m}]$ & -0.34839 & -0.34839 & -0.9311 \\
$\phi_{F 0}[\mathrm{rad}]$ & 4.3632 & 4.3632 & 4.3738 \\
$\theta[\mathrm{rad}]$ & 0.0981 & 0.0981 & 0.0983 \\
$\psi[\mathrm{rad}]$ & 0.048446 & 0.048446 & 0.04291 \\
$u[\mathrm{~m} / \mathrm{s}]$ & 217.05 & 217.05 & 218.15 \\
$v[\mathrm{~m} / \mathrm{s}]$ & $\ldots$ & 0.14795 & -0.2058 \\
$w[\mathrm{~m} / \mathrm{s}]$ & $\ldots$ & -0.14458 & -1.3333 \\
$p_{F 0}[\mathrm{rad} / \mathrm{s}]$ & $\ldots$ & 11.513 & 13.015 \\
$q[\mathrm{rad} / \mathrm{s}]$ & $\ldots$ & -2.1433 & -3.7745 \\
$r[\mathrm{rad} / \mathrm{s}]$ & $\ldots$ & -0.46654 & -0.7909 \\
\hline
\end{tabular}


Table 3 Initial and final aerodynamic parameter estimates

\begin{tabular}{cccc}
\hline Parameter & Base & Adjustment & Final \\
\hline$C_{X 0}$ & 0.33265 & 1.029 & 0.34230 \\
$C_{M 0}$ & 0.066235 & -1.0068 & -0.006669 \\
$C_{N 0}$ & 0.066235 & 0.38012 & 0.025177 \\
$C_{M A}$ & -11.5 & 0.6182 & -7.1093 \\
$C_{L 0}$ & 0.366652 & 0.27579 & 0.10112 \\
\hline
\end{tabular}

The process was repeated for $2.0 \leq t \leq 2.35$ (as depicted in Fig. 10). Static trimming to set the initial Euler angles, and Cartesian position were necessary for a good first guess. Since the roll-rate sensor was saturated, initial roll rate had to be estimated from the frequency of magnetometer oscillations.

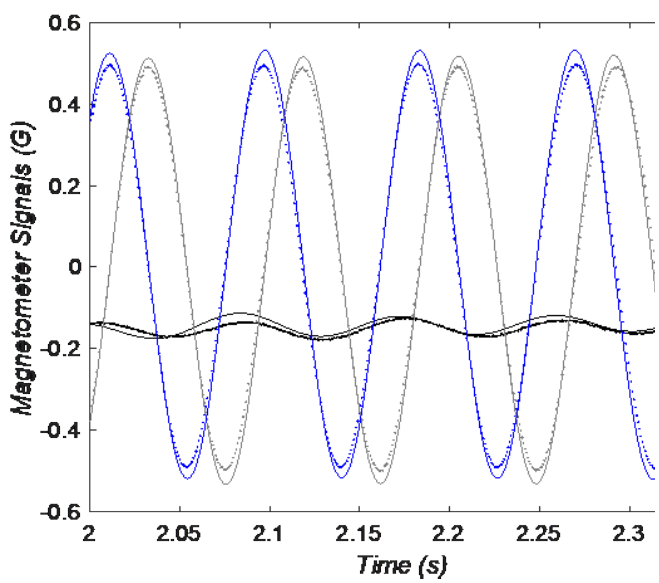

a) Magentometer

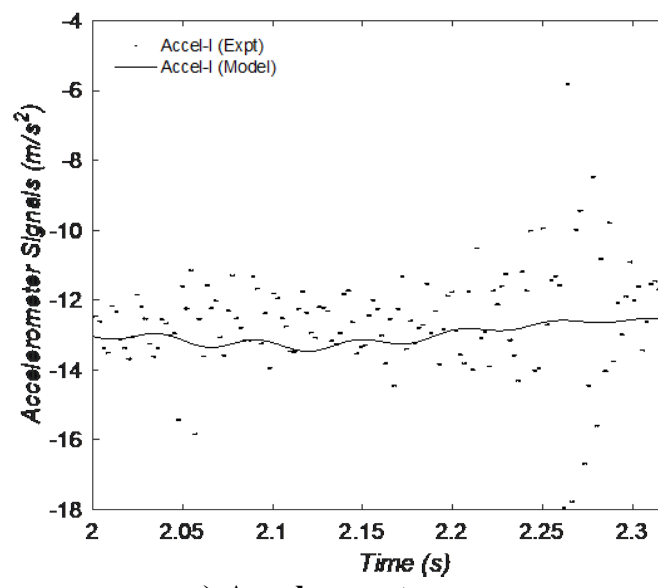

c) Accelerometer

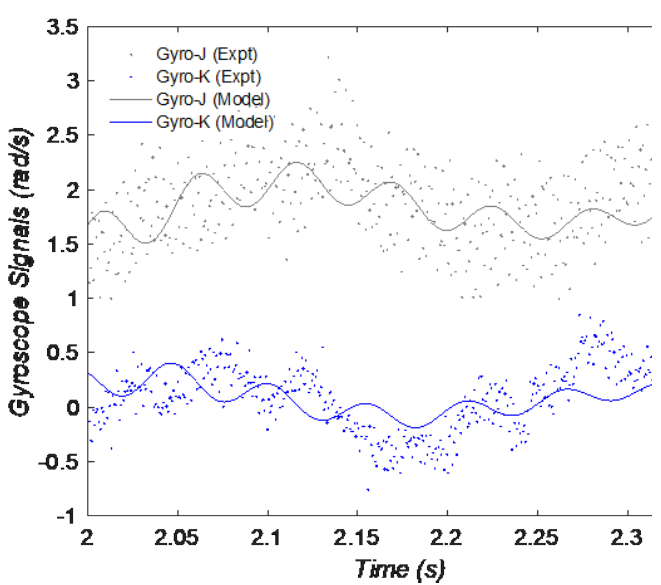

b) Gyroscope

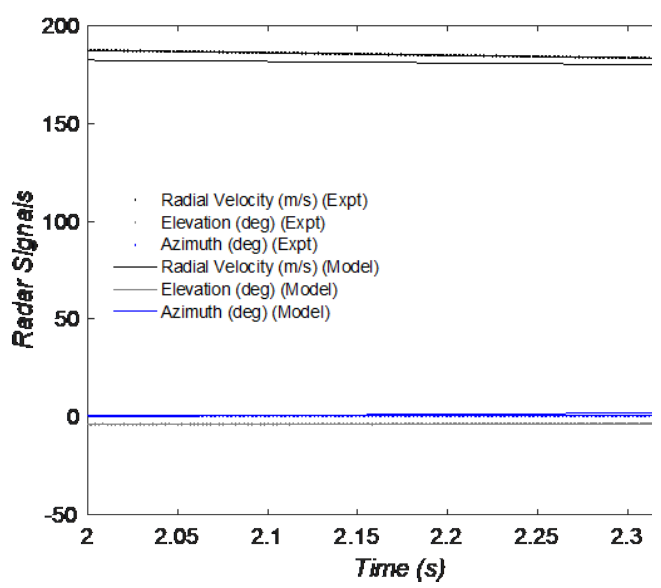

d) Radar

Fig. 10 In repeated process, model predictions and actual measurements for HMA Flight 8 data 
Roll-moment coefficients could not be adjusted without reference to the roll rate. Attempts to do so resulted in a singularity. Figure 10 compares the model predictions with the measurements used to refine the model over this phase of flight. The magnetometer I-axis prediction is slightly out of phase with the actual. The transverse magnetometer axes predictions have amplitudes slightly smaller than the data. Radar and I axis accelerometer signals match well. Tables 4 and 5 record the initial and refined initial condition and aerodynamic parameters for the second phase of flight.

Table 4 IC estimates from reducing actual HMA data, second phase of flight

\begin{tabular}{cccc}
\hline State & “Static" models & $\begin{array}{c}\text { Guesses entered } \\
\text { into NLLS }\end{array}$ & $\begin{array}{c}\text { Result of NLLS } \\
\text { after 15 iterations }\end{array}$ \\
\hline$x[\mathrm{~m}]$ & 422.15 & 420 & 425.19 \\
$y[\mathrm{~m}]$ & 2.0813 & 1.6268 & -2.706 \\
$z[\mathrm{~m}]$ & -38.636 & -34.423 & -28.529 \\
$\phi_{F 0}[\mathrm{rad}]$ & 5.1124 & 5.1124 & 5.1358 \\
$\theta[\mathrm{rad}]$ & 0.0343 & 0.0343 & 0.026439 \\
$\psi[\mathrm{rad}]$ & 0.34681 & 0.34681 & 0.30868 \\
$u[\mathrm{~m} / \mathrm{s}]$ & 217.05 & 187.39 & 190.03 \\
$v[\mathrm{~m} / \mathrm{s}]$ & $\ldots$ & -1.1111 & -0.49713 \\
$w[\mathrm{~m} / \mathrm{s}]$ & $\ldots$ & -3.0191 & -4.8174 \\
$p_{F 0}[\mathrm{rad} / \mathrm{s}]$ & $\ldots$ & 73.145 & 73.085 \\
$q[\mathrm{rad} / \mathrm{s}]$ & $\ldots$ & 1.8966 & 2.2518 \\
$r[\mathrm{rad} / \mathrm{s}]$ & $\ldots$ & 0 & -0.31993 \\
\hline
\end{tabular}

Table 5 Initial and final aerodynamic parameter estimates, second phase of flight

\begin{tabular}{cccc}
\hline Parameter & Base & Adjustment & Final \\
\hline$C_{X 0}$ & 0.33265 & 1.0464 & 0.34808 \\
$C_{N \alpha}$ & 9.847 & 0.19603 & 1.93031 \\
$C_{M 0}$ & 0.066235 & 5.6356 & 0.37327 \\
$C_{N 0}$ & 0.066235 & -5.5102 & -0.36500 \\
$C_{M A}$ & -11.5 & 0.83217 & -9.5700 \\
\hline
\end{tabular}

The process was repeated once more for $4.0 \leq t \leq 4.35$ (as depicted in Fig. 11). Static trimming to set the initial Euler angles and Cartesian position were necessary for a good first guess. Trimming the Euler angles to match the initial magnetometer readings resulted in the values shown in Table 6 . The initial roll angle is smaller 
than that shown in Table 4, as the algorithm will find $\phi$ within $0 \leq \phi<2 \pi$ and thus "unwrap" roll that may have accumulated between data segments. Since the rollrate sensor was saturated, initial roll rate had to be estimated from the frequency of magnetometer oscillations.

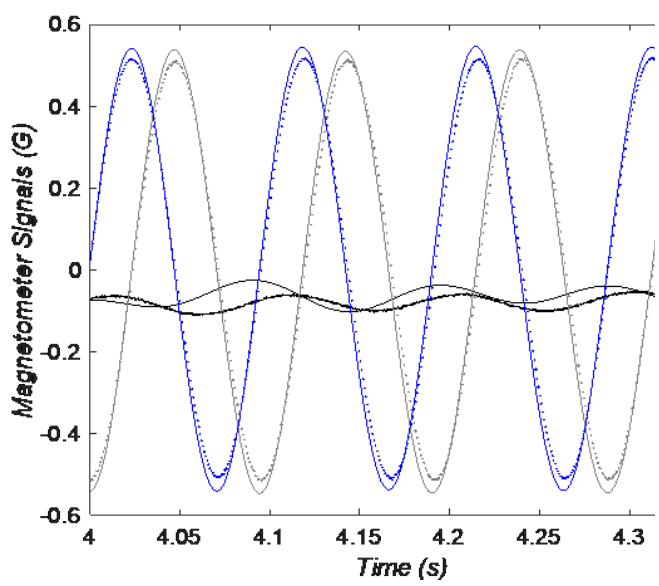

a) Magentometer

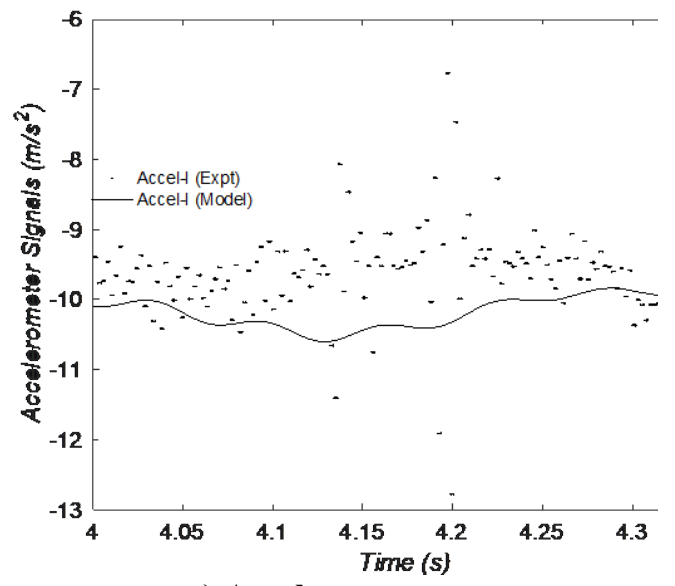

c) Accelerometer

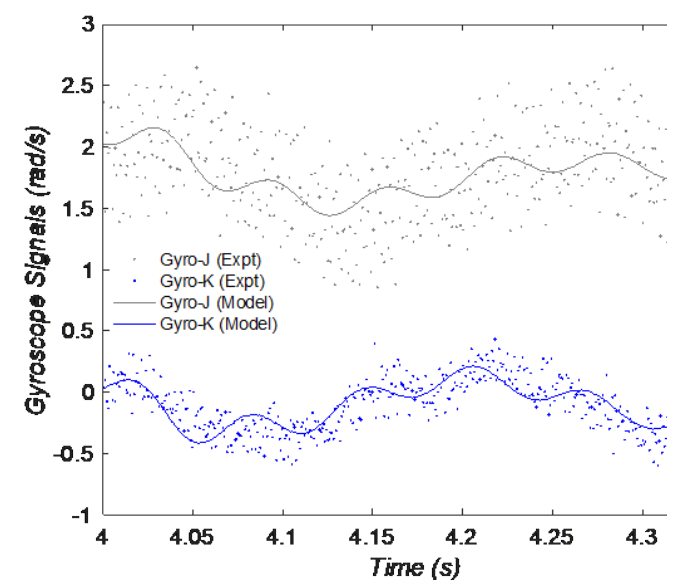

b) Gyroscope

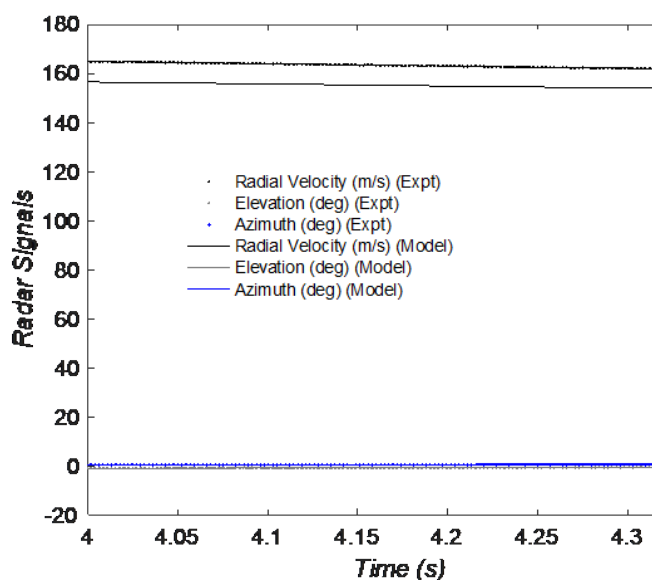

d) Radar

Fig. 11 Process repeated again, model predictions and actual measurements for HMA Flight 8 data

Roll-moment coefficients could not be adjusted without reference to the roll rate. Attempts to do so resulted in a singularity. $C_{N \alpha}$ and $C_{M A}$ were held constant at the values found from the second data segment. Figure 11 compares the model predictions with the measurements used to refine the model over this phase of flight. The magnetometer I-axis prediction is slightly out of phase with the actual. The transverse magnetometer axes predictions have amplitudes slightly smaller than the data. Radar and I-axis accelerometer signals match well. Transverse gyroscope axis predictions capture the slow pitch-yaw oscillation apparent in the data. The faster oscillation in the prediction is due to nontrivial estimates of 
gyroscope misalignment, causing the model to include a projection of body roll rate in the sensed pitch and yaw. This phenomenon is not obvious in the data due to large noise. Table 6 shows the IC estimates as they are refined through the various stages of model fidelity. Table 7 shows the estimated aerodynamic parameters for this phase.

Table 6 IC estimates from reducing actual HMA data, third phase of flight

\begin{tabular}{cccc}
\hline State & "Static" models & $\begin{array}{c}\text { Guesses entered } \\
\text { into NLLS }\end{array}$ & $\begin{array}{c}\text { Result of NLLS } \\
\text { after 15 iterations }\end{array}$ \\
\hline$x[\mathrm{~m}]$ & 857.72 & 857.72 & 863.46 \\
$y[\mathrm{~m}]$ & 3.3191 & 3.31911 & 2.0647 \\
$z[\mathrm{~m}]$ & -66.751 & -66.751 & -16.485 \\
$\phi_{F 0}[\mathrm{rad}]$ & 4.453 & 4.453 & 4.4502 \\
$\theta[\mathrm{rad}]$ & -0.1673 & -0.1673 & -0.16387 \\
$\psi[\mathrm{rad}]$ & 0.15901 & 0.15901 & 0.14712 \\
$u[\mathrm{~m} / \mathrm{s}]$ & 164.87 & 164.0 & 160.84 \\
$v[\mathrm{~m} / \mathrm{s}]$ & $\ldots$ & -1.1111 & -1.5113 \\
$w[\mathrm{~m} / \mathrm{s}]$ & $\ldots$ & -3.0191 & -2.789 \\
$p_{F 0}[\mathrm{rad} / \mathrm{s}]$ & $\ldots$ & 67 & 66.977 \\
$q[\mathrm{rad} / \mathrm{s}]$ & $\ldots$ & 1.8966 & 2.0262 \\
$r[\mathrm{rad} / \mathrm{s}]$ & $\ldots$ & 0 & -0.04602 \\
\hline
\end{tabular}

Table 7 Initial and final aerodynamic parameter estimates, third phase of flight

\begin{tabular}{cccc}
\hline Parameter & Base & Adjustment & Final \\
\hline$C_{X 0}$ & 0.33265 & 1.1296 & 037576. \\
$C_{M 0}$ & 0.066235 & 1.7526 & 0.11608 \\
$C_{N 0}$ & 0.066235 & -11.0241 & -0.73018 \\
\hline
\end{tabular}

\section{Conclusion}

This report has explored several methods to match onboard sensor and trackingradar data with models of varying fidelity. Throughout this study we have sought methods to improve the starting guesses of projectile initial states and thus enhance the ability of a nonlinear least squares algorithm to converge. Typically, models must be applied in order of increasing fidelity so that some of the states have very good starting guesses before the full 6-DOF model is applied. Recursive methods may suffer from unobservability, resulting in nonconvergence or even divergence of the model. Thus, batch methods are preferred when possible. The method was 
applied to data from an actual flight test. The algorithms found parameters in order to match the data well despite unmodeled sensor errors and the complete loss of some measurements due to saturation and other adverse effects. 


\section{References}

1. Burchett BT. Predictive optimal pulse-jet control for symmetric projectiles. Paper presented at: American Institute of Aeronautics and Astronautics SciTech Forum; 2014 Jan 13-17; National Harbor, MD.

2. Burchett B, Peterson A, Costello M. Prediction of the swerving motion of a dual-spin projectile with lateral pulse jets in atmospheric flight. Math Comp Model. 2002;35:812-834.

3. Van Loan C. Computing integrals involving the matrix exponential. IEEE Trans Auto Con. 1978;23(3):395-404.

4. McCoy RL. Modern exterior ballistics: the launch and flight dynamics of symmetric projectiles. Atglen (PA): Schiffer Military History; 1999.

5. Burchett BT. Unscented filtering for munition parameter estimation using onboard sensor telemetry. Paper presented at: American Institute of Aeronautics and Astronautics Aviation Forum; 2019 June 17-21; Dallas, TX.

6. Fresconi F, Celmins I, Silton S. Theory, guidance, and flight control for high maneuverability projectiles. Aberdeen Proving Ground (MD): Army Research Laboratory (US); 2014 Jan. Report No.: ARL-TR-6767.

7. Murphy CH. Data reduction for the free flight spark ranges. Aberdeen Proving Ground (MD): Ballistic Research Laboratories (US); 1954. BRL Report No. 900 .

8. Chapman GT, Kirk DB. A method for extracting aerodynamic coefficients from free-flight data. AIAA J. 1970;8(4):753-758.

9. Whyte RH, Jeung A, Bradley W. Chapman-Kirk reduction of free-flight range data to obtain nonlinear aerodynamic coefficients. Aberdeen Proving Ground (MD): Ballistic Research Laboratories (US); 1973 May. Report No.: 2298.

10. Hathaway W, Whyte R. Aeroballistic research facility free flight data analysis using the maximum likelihood method. Eglin Air Force Base (FL): Air Force Armament Laboratory (US); 1979 Dec. Report No.: AFATL-79-98.

11. Davis B, Hathaway W, Hathaway A, Thompson R. Extending telemetry reduction to aerodynamic coefficients and trajectory reconstruction (EXTRACTR) flight experiment using 155-mm M483A1 projectiles. Aberdeen Proving Ground (MD): Army Research Laboratory (US); 2005 Aug. Report No.: ARL-TR-3563. 
12. Fresconi F, Harkins T. Aerodynamics of a 120-mm mortar with a guidance kit obtained from telemetry experiments. Aberdeen Proving Ground (MD): Army Research Laboratory (US); 2008 Sep. Report No.: ARL-TR-4569.

13. Fresconi F, Harkins T. Experimental flight characterization of asymmetric and maneuvering projectiles from elevated gun firings. J Space Rockets. 2012;49(6):1120-1130.

14. Davis B, Condon J, Muller P. An instrumented 81-mm mortar target platform for dynamic engagements of high energy laser weapons. In: Woodley C, Cullis I, editors. Proceedings of the 29th International Symposium on Ballistics; 2016 May 9-13; Edinburgh, Scotland. Lancaster (PA): DESTech Publications, Inc. 


\section{List of Symbols, Abbreviations, and Acronyms}

\begin{tabular}{|c|c|}
\hline 3-D & 3-dimensional \\
\hline CG & center of gravity \\
\hline DOF & degrees of freedom \\
\hline EXTRACTR & $\begin{array}{l}\text { Extending Telemetry Reduction to } \\
\text { Coefficients and Trajectory Reconstruction }\end{array}$ \\
\hline HMA & High Maneuverability Airframe \\
\hline IC & initial condition \\
\hline NLLS & nonlinear least squares \\
\hline RHS & right-hand side \\
\hline UKF & Unscented Kalman Filter \\
\hline wrt & with respect to \\
\hline$\{x, y, z\}$ & projectile cg position in gun tube frame $[\mathrm{m}]$ \\
\hline$\left\{V_{x}, V_{y}, V_{z}\right\}$ & projectile cg velocity in gun tube frame $[\mathrm{m} / \mathrm{s}]$ \\
\hline$\{\phi, \theta, \psi\}$ & projectile roll, pitch and yaw in gun tube frame [rad] \\
\hline$\{u, v, w\}$ & projectile linear velocity in the body or no-roll frame $[\mathrm{m} / \mathrm{s}]$ \\
\hline$\{p, q, r\}$ & projectile angular rates in the body or no-roll frame $[\mathrm{rad} / \mathrm{s}]$ \\
\hline$\{X, Y, Z\}$ & total force vector in projectile body frame $[\mathrm{N}]$ \\
\hline$\{L, M, N\}$ & total moment vector in projectile body frame $[\mathrm{N}-\mathrm{m}]$ \\
\hline$m$ & projectile mass $[\mathrm{kg}]$ \\
\hline$T$ & rotation matrix \\
\hline $\mathbf{S}$ & skew matrix for cross product \\
\hline $\mathbf{I}$ & inertia matrix $\left[\mathrm{kg}-\mathrm{m}^{2}\right]$ or identity matrix \\
\hline$\left\{R_{V}, R_{\theta}, R_{\psi}\right\}$ & radar measurement vector \\
\hline$\left\{\tilde{m}_{E x}, \tilde{m}_{E y}, \tilde{m}_{E z}\right\}$ & earth magnetic field measured in body frame $[\mathrm{nT}]$ \\
\hline$\left\{\tilde{a}_{x}, \tilde{a}_{y}, \tilde{a}_{z}\right\}$ & accelerometer measurements in body frame $\left[\mathrm{m} / \mathrm{s}^{2}\right]$ \\
\hline$\hat{C}_{X}$ & dimensional drag coefficient $=\rho \pi D^{2} C_{X 0} /(8 m)[1 / \mathrm{m}]$ \\
\hline
\end{tabular}




$\begin{array}{ll}V & \text { total velocity } \sqrt{u^{2}+v^{2}+w^{2}}[\mathrm{~m} / \mathrm{s}] \\ \rho & \text { atmospheric density }\left[\mathrm{kg} / \mathrm{m}^{3}\right] \\ \mathbf{S} & \text { area of projectile cross section }\left[\mathrm{m}^{2}\right] \\ S & \text { arclength traveled [calibers] } \\ D & \text { projectile diameter [m] } \\ C_{X 0} & \text { zero angle of attack coefficient } \\ C_{N A} & \text { normal force due to angle of attack coefficient } \\ C_{M A} & \text { pitch moment due to AOA coefficient } \\ C_{L 0} & \text { static roll moment coefficient } \\ C_{M Q} & \text { pitch damping coefficient } \\ C_{L p} & \text { roll damping coefficient } \\ \text { Subscript } & \\ b 2 i & \text { ground to body frame } \\ b 2 e & \text { body to Euler frame } \\ F & \text { forward body } \\ A & \text { aft body } \\ p & \text { particular (forced) solution } \\ \text { Superscript } & \\ T & \text { matrix transpose } \\ & \end{array}$




$\begin{array}{cl}1 & \text { DEFENSE TECHNICAL } \\ \text { (PDF) } & \text { INFORMATION CTR } \\ & \text { DTIC OCA } \\ 1 & \text { CCDC ARL } \\ \text { (PDF) } & \text { FCDD RLD CL } \\ & \text { TECH LIB } \\ 1 & \text { GOVT PRINTG OFC } \\ (\mathrm{PDF}) & \text { A MALHOTRA } \\ 1 & \text { RHIT } \\ \text { (PDF) } & \text { B BURCHETT } \\ & \text { CCDC ARL } \\ 6 & \text { FCDD RLW LE } \\ \text { (PDF) } & \text { P WEINACHT } \\ & \text { V A BHAGWANDIN } \\ & \text { I CELMINS } \\ & \text { J DESPIRITO } \\ & \text { L D FAIRFAX } \\ & \text { F E FRESCONI }\end{array}$

